\title{
Article \\ Formal Chaos Existing Conditions on a Transmission Line Circuit with a Piecewise Linear Resistor
}

\author{
Kazuya Ozawa ${ }^{1, *(\mathbb{D})}$, Kaito Isogai ${ }^{1}(\mathbb{D})$, Hideo Nakano ${ }^{2}$ and Hideaki Okazaki $^{1}$ \\ 1 Graduate School of Electrical and Information Engineering, Shonan Institute of Technology, \\ 1-1-25 Tsujido Nishikaigan, Fujisawa 251-8511, Japan; kaito.isogai.3@gmail.com (K.I.); \\ okazaki@sc.shonan-it.ac.jp (H.O.) \\ 2 Private Consultant, Fujisawa 251-8511, Japan; nanma17@nifty.com \\ * Correspondence: kazuya.ozawa.77@gmail.com
}

Citation: Ozawa, K.; Isogai, K.; Nakano, H.; Okazaki, H. Formal Chaos Existing Conditions on a Transmission Line Circuit with a Piecewise Linear Resistor. Appl. Sci. 2021, 11, 9672. https://doi.org/ 10.3390/app11209672

Academic Editor: Luigi Fortuna

Received: 1 September 2021

Accepted: 12 October 2021

Published: 17 October 2021

Publisher's Note: MDPI stays neutral with regard to jurisdictional claims in published maps and institutional affiliations.

Copyright: (C) 2021 by the authors. Licensee MDPI, Basel, Switzerland. This article is an open access article distributed under the terms and conditions of the Creative Commons Attribution (CC BY) license (https:/ / creativecommons.org/licenses/by/ $4.0 /)$.

\begin{abstract}
By using one-dimensional (1-D) map methods, some lossless transmission line circuits with a short at one side terminal have been actively studied. Bifurcation results or chaotic states in the circuits have been reported. On the other hand, many weak or strong definitions such that a 1-D map is mathematically chaotic are still being studied. In such definitions, the definition of formal chaos is well known as being the most traditional and most definite. However, formal chaos existences have not been rigorously proven in such circuits. In this paper, a general lossless transmission circuit is considered first with a dc bias voltage source in series with a load resistor at one side terminal and with a three-segment piecewise linear resistor at another side terminal. Secondly, the method for deriving a 1-D map describing the behavior of the circuit is summarized. Thirdly, to provide a basis of chaotic application for the 1-D map, the mathematical definition of formal chaos and the sufficient conditions of the existence of formal chaos are discussed. Furthermore, by using Maple, formal chaos existences and bifurcation behavior of 1-D maps are presented. By using the Lyapunov exponent, the observability of formal chaos in such bifurcation processes is outlined. Finally, the principal results and the future works are summarized.
\end{abstract}

Keywords: formal chaos; shift dynamics $\left(\sigma, \Sigma_{2}\right)$ with 2 symbols; Lyapunov exponent; lossless transmission line circuit; piecewise linear resistor

\section{Introduction}

Transmission line circuits, switched capacitor circuits, neuron model circuits, and constrained circuits are notable as the nonlinear circuits of which behavior may be described by one-dimensional (1-D) maps [1-11]. Furthermore, in [12], by using real imperfect integrated devices induced by unavoidable manufacturing imperfections that plausibly have so called hidden dynamics with parasitic effects and nonidealities, the possibility of designing imperfect electronic circuits generating megahertz chaotic oscillations, without the use of additional capacitors or inductors, has been explored and discussed.

By using one-dimensional (1-D) map methods, some lossless transmission line circuits with a short at one side terminal [5-11] (or, with a dc bias voltage source in series with a load resistor at one side terminal [7]) have been actively studied. On the other hand, many weak or strong definitions such that a 1-D map is mathematically chaotic are still being studied [13-18]. In this paper, we use the following definition that is well known as most traditional and most definite since Moser [19].

Definition 1. A 1-D discrete dynamical system or 1-D map $\varphi$ on $M$ is said to be chaotic if there exists an invariant subset $\Lambda \subset M$ on which some iterates of $\varphi$ is topologically conjugate to the shift dynamics $\left(\sigma, \Sigma_{m}\right)$ with $m$ symbols. 
The shift dynamics $\left(\sigma, \Sigma_{m}\right)$ with $\mathrm{m}$ symbols has the following properties [13,15-17]: (1) There are countably many periodic points with different periods in $\Sigma_{m}$. (2) The set of all periodic points in dense in $\Sigma_{m}$. (3) $\sigma$ is topologically transitive. Namely, there exists a point $c \in \Sigma_{m}$ where its orbit is dense in $\Sigma_{m}$. (4) $\sigma$ is topologically expansive. (5) $\Sigma_{m}$ is homeomorphic to a Cantor set. (6) The topological entropy of $\sigma$ is $h(\sigma)=\log _{e} m$.

For a 1-D discrete dynamical system $\varphi$, it is well known that " $\varphi$ has a homoclinic point" implies Definition 1, and they are often equivalent $[15,20,21]$. Definition 1 is not based on the theoretical measure but is based on the topological viewpoint, and it is called formal chaos or topological chaos, which may be not observable generally [13]. Thus, we call the chaos in Definition 1 formal chaos.

Binary sequences based on observable chaotic behavior produced by 1-D maps coming from the nonlinear circuits are reported and well known to have good statistical properties useful for applications relative to several digital communication systems $[4,13]$. However, the sufficient conditions for the existence of observable chaos are so strong that most 1-D maps coming from general nonlinear circuits cannot be rigorously proved to possess observable chaos. Indeed, for general 1-D maps such that the absolute slops of them are either greater or less than unity, it is very difficult to rigourously prove the existence of observable chaos, except for 1-D maps such as the logistic map family or 1-D piecewise linear maps such that all their absolute slopes of are greater than unity.

Once the existence of formal chaos is guaranteed, the degree of the observability of the chaos can be checked by the existence of maximal positive Lyapunov exponent [14] through computer simulations from the viewpoint of engineering. Hence, we pay attention to the sufficient condition that formal chaos in Definition 1 exists in a 1-D map.

In $[5,6]$, a lossless transmission line circuit with a short at one side terminal and with a three-segment piecewise linear (3SPWL) resistor (so called Nagumo's 3SPWL resistor) at another side terminal is considered. The $v-i$ characteristic of the (3SPWL) resistor is not only in point symmetry at the origin but also in all four quadrants of a $(v, i)$ coordinate system. The method for deriving a 1-D map describing the dynamics of the circuit is discussed at the base of the incident and reflected waves. Some analytical results are obtained by using the 1-D map. In [8-10], for a lossless transmission line circuit with a short at one side terminal and with a three-segment piecewise linear resistor function at another side terminal, the conditions are provided for the existence of the explicit function form of the incident and reflected wave transformed from the three-segment piecewise linear resistor function, the existence of the 1-D map, and the existence of the invariant interval of the 1-D map. Furthermore, in [8-11], by using a 1-D map, analytical bifurcation results or chaotic states (except for formal chaos) are reported in terms of numerical simulation.

However, formal chaos and observable chaos existence $[13,18,20]$ have not been rigorously proven in such lossless transmission line circuits in [5-11]. In [22], by using interval arithmetic, the formal chaos existence was proven in a lossless transmission line circuit, but the proof is very tedious.

In this paper, from the above background, in Section 2, we consider a lossless transmission circuit with a dc bias voltage source in series with a load resistor at one side terminal and with a three-segment piecewise linear resistor at another side terminal. In Section 3, we summarize the method for deriving a 1-D map describing the behavior of the circuit. In Section 4, in order to provide a basis of chaotic application for the 1-D map (or the transmission circuit), we discuss the mathematical definition of formal chaos and the sufficient conditions of the existence of formal chaos for generating 1-D maps. In Section 5, using Maple [23], we present an example of formal chaos existence and several examples of bifurcation behavior of 1-D maps. Using the degree of observability of chaotic states in terms of Lyapunov exponent, we discuss the observability of formal chaos in such bifurcation processes. In the Conclusion section, we summarize the principal results and the future works. In particular, we mention the possibility of designing imperfect transmission lines with parasitic effects and nonidealities inside real integrated devices generating high frequency ranges and chaotic oscillations without the use of additional capacitors or inductors. 


\section{Lossless Transmission Circuit Equations with Terminal Conditions}

Incorporating the works in [5-10], we consider a lossless transmission circuit with a dc bias voltage source in series with a load resistor at one side terminal and with a three-segment piecewise linear resistor at another side terminal such as $(\mathrm{N})$ Nagumo's 3SPWL resistor in Figures 1 and 2 or (T) the present authors' Modeled Tunnel Diode (TD) 3SPWL resistor in Figures 3 and 4 in Figure 5. The circuit in Figure 5 can be represented by the circuit in Figure 6 with 3SPWL resistor $(\mathrm{N})$ or $(\mathrm{T})$ together with the side terminal conditions as follows. At one side terminal $A$ of the circuit, we have the following: (i) a dc bias voltage source in series with 3SPWL resistor $(\mathrm{N})$ or $(\mathrm{T}): E_{B 0} \neq 0$; and (ii) 3SPWL resistor $(\mathrm{N})$ or $(\mathrm{T}): E_{B 0}=0$. At another side terminal $B$ of the circuit, we have the following: (1) short: $E_{B}=0, R_{L}=0$; (2) open: $E_{B}=0, R_{L} \rightarrow \infty$; (3) a load resistor: $E_{B}=0, R_{L} \neq 0$; (4) a dc bias voltage source: $E_{B} \neq 0, R_{L}=0$; or (5) a dc bias voltage source in series with a load resistor: $E_{B} \neq 0, R_{L} \neq 0$. We focus on considering a lossless transmission line circuit with 3SPWL resistor $(\mathrm{N})$ or $(\mathrm{T})$, together with the above side terminal conditions in Figure 6.
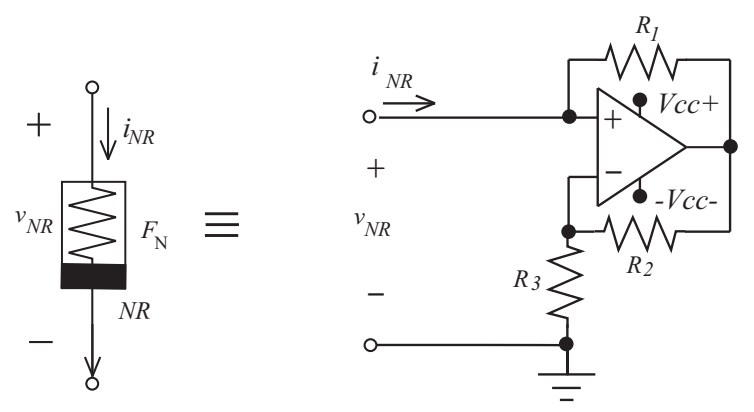

Figure 1. Op amp circuit based Nagumo's 3SPWL resistor.

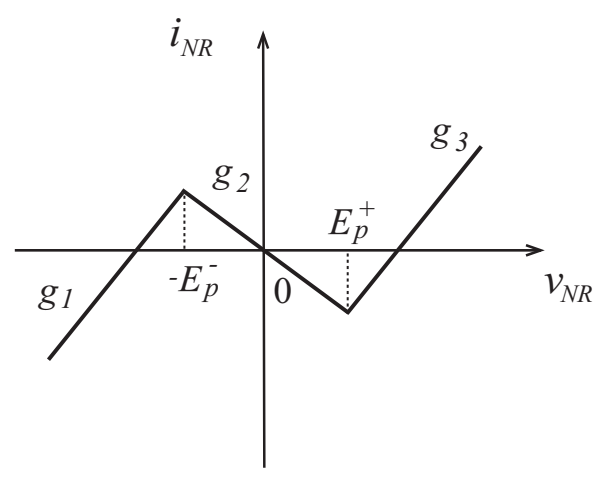

Figure 2. $v_{N R}-i_{N R}$ characteristic of Nagumo's 3SPWL resistor.

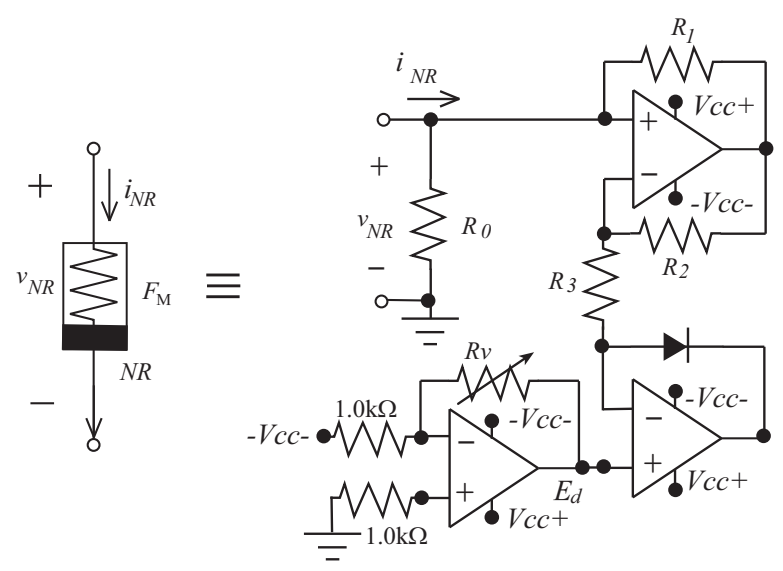

Figure 3. Op amp circuit based Modeled Tunnnel Diode 3SPWL resistor. 


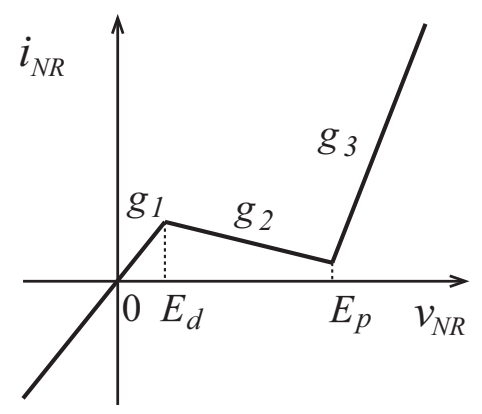

Figure 4. $v_{N R}-i_{N R}$ characteristic of Modeled Tunnnel Diode 3SPWL resistor.
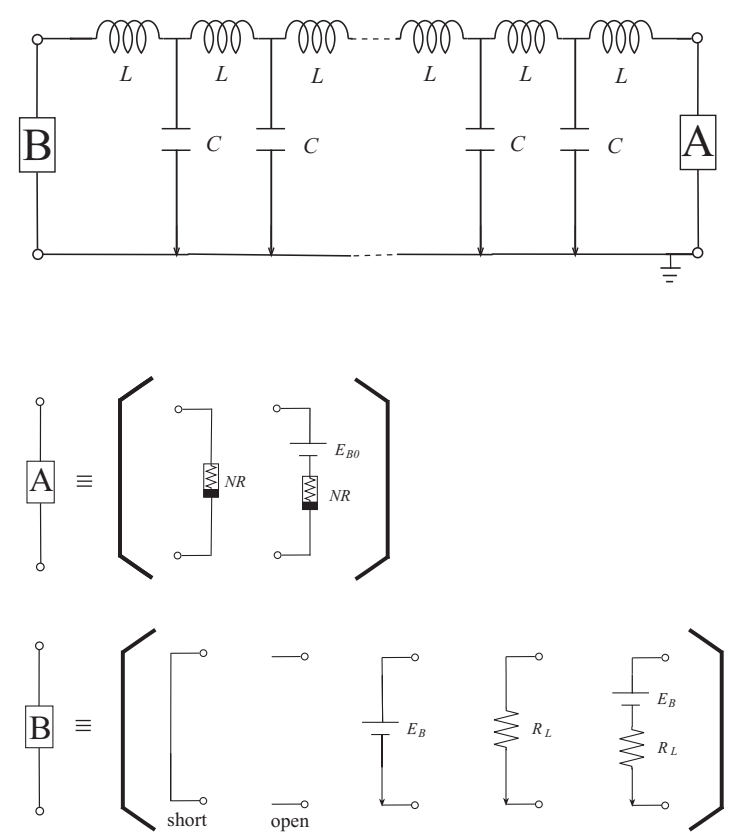

Figure 5. Transmission line circuit with generally practical side terminal conditions.

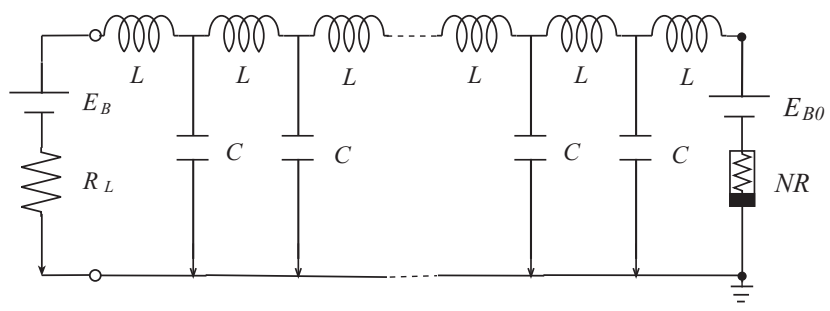

Figure 6. Transmission line circuit with standard side terminal conditions.

\subsection{Three-Segment Piecewise Linear Resistor}

The three-segment Piecewise Linear (3SPWL) resistor $i_{N R}=F_{k}\left(v_{N R}\right),(k=N, T)$ in Figure 5 is realized by the op amp based circuit $(N)$ Nagumo's 3SPWL resistor in Figure 1 and Figure 2 or (T) the present authors' Modeled Tunnel Diode (TD) 3SPWL resistor in Figures 3 and 4 . In Figure 1 or Figure $3, E_{s}^{+}$(or $-E_{s}^{-}$) denotes positive (or negative) saturation of the op amp, and $V_{c c}^{+}\left(\right.$or $\left.-V_{c c}^{-}\right)$denotes positive (or negative) power supply. $E_{d}$ in Figure 3 denotes the forward voltage drop of the ideal switching diode. In Figure 2 or Figure 4 , the $v-i$ characteristic of $F_{k}$ is always of the $N$ type such that $g_{1}, g_{2}$, and $g_{3}$ are positive, negative, and positive slopes, respectively. 
(N) Nagumo's 3SPWL Resistor $F_{N}$ :

The slopes $g_{i}, i=1, \cdots, 3$ and break points $-E_{p}^{-}$and $E_{p}^{+}$in Figure 2 are given as follows:

$$
\begin{aligned}
g_{1} & =\frac{1}{R_{1}}, g_{2}=-\frac{R_{1}}{R_{2} R_{3}}=-\frac{\zeta}{R_{1}}, g_{3}=\frac{1}{R_{1}}, \\
E_{p}^{-} & =\frac{E_{s}^{-} R_{3}}{R_{2}+R_{3}}, E_{p}^{+}=\frac{E_{s}^{+} R_{3}}{R_{2}+R_{3}}=\eta E_{p}^{-},
\end{aligned}
$$

on the condition that $\eta>-1, \zeta>0, E_{p}^{+}>0>-E_{p}^{-}$, and $R_{3}=\frac{R_{1}^{2}}{\zeta R_{2}}$. $E_{p}^{-}$is freely set up by $V_{c c}^{-} \cdot \eta$ (i.e., $E_{p}^{+}$) is also freely set up by $V_{c c}^{+}$because, for any op amps, $E_{s}^{+}$(or $-E_{s}^{-}$) is generally in proportion to $V_{c c}^{+}\left(\right.$or $\left.-V_{c c}^{-}\right)$.

(T) TD 3SPWL Resistor $F_{T}$ :

The slopes $g_{i}, i=1, \cdots, 3$ and break points $E_{d}$ and $E_{p}$ in Figure 4 are given as follows:

$$
\begin{aligned}
& g_{1}=\frac{1}{R_{0}}, g_{2}=\frac{1}{R_{0}}-\frac{R_{1}}{R_{2} R_{3}}=-\frac{\zeta}{R_{0}}, \\
& g_{3}=\frac{1}{R_{0}}+\frac{1}{R_{1}}=\frac{2}{R_{0}}, E_{p}=\frac{E_{s}^{+} R_{3}}{R_{2}+R_{3}}=\eta E_{d},
\end{aligned}
$$

on the conditions that $\eta>1, \zeta>0, E_{p}>E_{d}>0, R_{3}=\frac{R_{0} R_{1}}{R_{2}(\zeta+1)}$, and $R_{1}=R_{0}$. $E_{d}$ is freely set up by $R_{V} \cdot \eta$ (i.e., $E_{p}$ ) is also freely set up by $V_{c c}^{+}$because, for any op amps, $E_{s}^{+}$is generally in proportion to $V_{c c}^{+}$.

\subsection{Transmission Line Circuit Equation}

The relation of the voltage $v$ and the current $i$ in the line in Figure 6 becomes the following:

$$
\frac{\partial v}{\partial \tau}=-\frac{\partial i}{\partial x}, \frac{\partial i}{\partial \tau}=-\frac{\partial v}{\partial x},
$$

where $Z=\sqrt{\frac{L}{C}}$ is the characteristic impedance of the line; $s=\frac{1}{\sqrt{L C}}$ is the propagation velocity of waves in the line; $T=\frac{2 l}{s}, v=v_{C} / V_{\max }, i=i_{L} / I_{\max }, x=x_{l} / 2 l, \tau=t / T, L$ are the series of inductance per unit length of the line; $C$ is the parallel capacitance per unit length of the line; $l$ is line length; $v_{C}$ is the voltage in the line; $i_{L}$ is the current in the line; $x_{l}$ is the coordinate in the line; $v$ is the non-dimensional voltage in the line; $i$ is the non-dimensional current in the line; $x$ is the non-dimensional coordinate in the line; and $\tau$ is non-dimensional time. Accordingly, by eliminating $i$ (or $v$ ), we have the following.

$$
\frac{\partial^{2} v(\tau, x)}{\partial \tau^{2}}=\frac{\partial^{2} v(\tau, x)}{\partial x^{2}},\left(\frac{\partial^{2} i(\tau, x)}{\partial \tau^{2}}=\frac{\partial^{2} i(\tau, x)}{\partial x^{2}}\right) .
$$

The following Equations (3) and (4) provide the boundary conditions of Figure 6. Equations (3) and (4) also provide the boundary conditions of Figure 5 as follows. At one side terminal, we have the following: (i) a dc bias voltage source in series with 3SPWL resistor $(\mathrm{N})$ or $(\mathrm{T}): \xi \neq 0$ and (ii) 3 SPWL resistor $(\mathrm{N})$ or $(\mathrm{T}): \xi=0$. At another side terminal, we have the following: (1) short: $E=0, R=0$; (2) open: $E=0, R \rightarrow \infty$; (3) a load resistor: $E=0, R \neq 0$; (4) a dc bias voltage source: $E \neq 0, R=0$; or (5) a dc bias voltage source in series with a load resistor: $E \neq 0, R \neq 0$.

$$
\begin{aligned}
& v(\tau, 0)=E-R i(\tau, 0) \\
& i\left(\tau, \frac{1}{2}\right)=f_{k}\left(v\left(\tau, \frac{1}{2}\right), \zeta, \eta, \xi\right),(k=N, T) .
\end{aligned}
$$




$$
f_{k}(v, \zeta, \eta, \xi)=I_{\max } F_{k}\left(V_{\max }\left(v-E_{0}\right)\right)=
$$

(N) Nagumo's 3SPWL Resistor $f_{N}$ :

$$
\begin{cases}\frac{v+(1-\xi) b_{p}}{r}+\frac{\zeta b_{p}}{r} & , v<-(1-\xi) b_{p} \\ -\frac{\zeta v}{r}+\frac{\zeta \xi b_{p}}{r} & ,-(1-\xi) b_{p} \leq v \\ \frac{\left(v-(\eta+\xi) b_{p}\right)}{r}-\frac{\zeta \eta b_{p}}{r} & ,(\eta+\xi) b_{p}\end{cases}
$$

Here, $\zeta>0, \eta>-1, r=\frac{R_{1}}{R_{\max }}(>0), b_{p}=\frac{E_{p}^{-}}{V_{\max }}(>0), \eta b_{p}=\frac{E_{p}^{+}}{V_{\max }}\left(>0>-b_{p}\right)$.

(T) TD 3SPWL Resistor $f_{T}$ :

$$
\begin{cases}\frac{v}{r}-\frac{\xi b_{p}}{r} & , v<(1+\xi) b_{p} \\ -\frac{\zeta\left(v-(1+\xi) b_{p}\right)}{r}+\frac{b_{p}}{r} & ,(1+\xi) b_{p} \leq v \\ \frac{2\left(v-(\eta+\xi) b_{p}\right)}{r}-\frac{\zeta(\eta-1) b_{p}}{r}+\frac{b_{p}}{r} & \leq(\eta+\xi) b_{p} \\ & ,(\eta+\xi) b_{p}<v\end{cases}
$$

Here, $\zeta>0, \eta>1, r=\frac{R_{1}}{R_{\max }}(>0), b_{p}=\frac{E_{d}}{V_{\max }}(>0), \eta b_{p}=\frac{E_{p}}{V_{\max }}\left(>b_{p}\right)$. In addition in $(\mathrm{N})$ or $(\mathrm{T}), \xi b_{p}=E_{0}, R=R_{L} / R_{\max }, E_{0}=E_{B 0} / V_{\max }, E=E_{B} / V_{\max }, E_{B 0}$, and $E_{B}$ are the dc bias voltage sources; $R_{L}$ is the load resistor; and $f_{k}(v, \zeta, \eta, \xi)$ is the non-dimensional current of 3SPWL resistor, $(k=N, T)$.

\section{1-D Map Describing the Behavior of the Transmission Line Circuit}

\subsection{Derivation of 1-D Map}

Here, we discuss how to derive the 1-D map or the difference equation of which the dynamics completely describes the dynamics of Equations (2)-(6).

The 1-D wave equation of Equation (2) has the d'Alembert's solution, which is described as the following.

$$
\begin{aligned}
& v(\tau, x)=\psi(\tau-x)-\phi(\tau+x) \\
& i(\tau, x)=\frac{\psi(\tau-x)+\phi(\tau+x)}{Z} .
\end{aligned}
$$

Equation (7) can be solved with regard to the scattering variables, that is, $\psi$ and $\phi$. Then, we have the following description.

$$
\left(\begin{array}{c}
\psi \\
\phi
\end{array}\right)=\frac{1}{\sqrt{2}}\left(\begin{array}{c}
\cos \left(-\frac{\pi}{4}\right)-Z \sin \left(-\frac{\pi}{4}\right) \\
\sin \left(-\frac{\pi}{4}\right) Z \cos \left(-\frac{\pi}{4}\right)
\end{array}\right)\left(\begin{array}{c}
v \\
i
\end{array}\right) .
$$

Equation (8) denotes that the $(\psi, \phi)$-coordinate system is identical with the $-\pi / 4$ rotation of $(v, i)$-coordinate system. Substituting Equation (7) into Equations (3) and (4), we rewrite the boundary conditions as follows:

$$
\begin{aligned}
\psi(\tau)-\phi(\tau) & =E-R \frac{(\psi(\tau)+\phi(\tau))}{Z}, \\
\frac{\psi(\tau-1)+\phi(\tau)}{Z} & =f_{k}((\psi(\tau-1)-\phi(\tau)), \zeta, \eta, \xi),
\end{aligned}
$$

where the time is shifted $1 / 2$ back in (10) and $(k=N, T)$.

The following Equations (9) and (10) provide the boundary conditions of Figure 6. Equations (9) and (10) also provide the boundary conditions of Figure 5 as follows. 
At one side terminal, we have the following: (i) a dc bias voltage source in series with 3SPWL resistor (N) or $(\mathrm{T}): \xi \neq 0$ and (ii) 3SPWL resistor $(\mathrm{N})$ or $(\mathrm{T}): \xi=0$. At another side terminal, we have the following: (1) short: $E=0, R=0$; (2) open: $E=0, R \rightarrow \infty$; (3) a load resistor: $E=0, R \neq 0$; (4) a dc bias voltage source: $E \neq 0, R=0$; or (5) a dc bias voltage source in series with a load resistor: $E \neq 0, R \neq 0$.

Since $f_{k}(v, \zeta, \eta, \xi)$ is a three segment piecewise linear function, we can identify Equation (11) with $f_{k}(v, \zeta, \eta, \xi)$ for $v \in I_{k w} \subset \mathcal{R}, w=1,2,3$ :

$$
\begin{aligned}
& m_{k w} v+n_{k w}, v \in I_{k w} \subset \mathcal{R}, m_{k w}, n_{k w} \in \mathcal{R}, w=1,2,3, \\
& I_{k w} \subset \mathcal{R},: \text { wth segment of three domain intervals, }
\end{aligned}
$$

where $m_{k w}:(\zeta, \eta, \xi) \rightarrow \mathcal{R}$ and $n_{k w}:(\zeta, \eta, \xi) \rightarrow \mathcal{R}$.

Here, from Equations (9) and (10), we briefly show how to derive an explicit piecewise linear 1-D map of $\psi(\tau)$ and $\psi(\tau-1)$. First of all, substitute Equation (11) into $f_{k}(v, \zeta, \eta, \xi)$ at the right hand side of Equation (10). We obtain Equations (12) and (13). Now since Equations (9) and (12) are simultaneous linear equations, we solve Equations (9) and (12) as Equation (14). We also solve Equations (12) and (13) as Equation (15).

$$
\begin{gathered}
\frac{\psi(\tau-1)+\phi(\tau)}{Z}=m_{k w}(\psi(\tau-1)-\phi(\tau))+n_{k w}, \\
\psi(\tau-1)-\phi(\tau) \in I_{k w} \subset \mathcal{R} . \\
\psi(\tau)=\frac{1-\frac{R}{Z}\left(\frac{m_{k w}-\frac{1}{Z}}{1+\frac{R}{Z}}\left(\frac{1}{m_{k w}+\frac{1}{Z}}(\tau-1)-\frac{n_{k w}}{m_{k w}+\frac{1}{Z}}+\frac{E}{1-\frac{R}{Z}}\right),\right.}{\psi(\tau-1) \in \frac{m_{k w}+\frac{1}{Z}}{\frac{2}{Z}}\left(I_{k w}+\frac{n_{k w}}{m_{k w}+\frac{1}{Z}}\right) \subset \mathcal{R},}
\end{gathered}
$$

In the following, using the similar procedure from Equations (9)-(15), we provide Theorem 1 and Corollary 1 such that $\phi(\tau)$ and $\psi(\tau)$ are explicitly determined by 1-D maps of $\psi(\tau-1)$ with the iterative time 1 . We also provide Corollary 2 such that $v\left(\tau+\frac{1}{2}, \frac{1}{2}\right)$ is explicitly determined by the1-D map of $v\left(\tau-\frac{1}{2}, \frac{1}{2}\right)$.

Theorem 1. If $1>\frac{r}{Z}>\zeta>0$ and Nagumo's 3SPWL Resistor $(k=N)$ is $\eta>-1$ or TD 3SPWL Resistor $(k=T)$ is $\eta>1$, then $\chi_{k}(k=N, T): \mathcal{R} \rightarrow \mathcal{R}$ is defined by the following:

$$
\begin{gathered}
\phi(\tau)=\chi_{k}(\psi(\tau-1))= \begin{cases}\chi_{k 1}(\psi(\tau-1)) & , \psi(\tau-1)<\psi_{k 1} \\
\chi_{k 2}(\psi(\tau-1)) & , \psi_{k 1} \leq \psi(\tau-1) \leq \psi_{k 2} \\
\chi_{k 3}(\psi(\tau-1)) & , \psi_{k 2}<\psi(\tau-1)\end{cases} \\
0<D \chi_{k 1}<1, D \chi_{k 2}<-1,0<D \chi_{k 3}<1
\end{gathered}
$$

which also holds when the following is the case:

$$
\begin{aligned}
\chi_{N 1}(\psi(\tau-1)) & =\frac{\left(1-\frac{r}{Z}\right) \psi(\tau-1)}{\frac{r}{Z}+1}+\frac{((1-\xi)+\zeta) b_{p}}{\frac{r}{Z}+1}, \\
\chi_{N 2}(\psi(\tau-1)) & =\frac{\left(\frac{\zeta}{Z}+\frac{r}{Z}\right) \psi(\tau-1)}{\left(\frac{\zeta}{Z}-\frac{r}{Z}\right)}-\frac{\zeta \xi b_{p}}{\left(\zeta-\frac{r}{Z}\right)}, \\
\chi_{N 3}(\psi(\tau-1)) & =\frac{\left(1-\frac{r}{Z}\right) \psi(\tau-1)}{\left(\frac{r}{Z}+1\right)}-\frac{(\eta(\zeta+1)+\xi) b_{p}}{\left(\frac{r}{Z}+1\right)}, \\
\psi_{N 1} & =\frac{1}{2}\left(-(1-\xi)+\frac{Z \zeta}{r}\right) b_{p}, \\
\psi_{N 2} & =\frac{1}{2}\left(\eta+\xi-\frac{Z \zeta \eta}{r}\right) b_{p}\left(>\psi_{N 1}\right),
\end{aligned}
$$


or we have the following:

$$
\begin{aligned}
\chi_{T 1}(\psi(\tau-1)) & =\frac{\left(1-\frac{r}{Z}\right) \psi(\tau-1)}{\left(\frac{r}{Z}+1\right)}-\frac{\xi b_{p}}{\left(\frac{r}{Z}+1\right)}, \\
\chi_{T 2}(\psi(\tau-1)) & =\frac{\left(\zeta+\frac{r}{Z}\right) \psi(\tau-1)}{\left(\zeta-\frac{r}{Z}\right)}-\frac{(\zeta(1+\xi)+1) b_{p}}{\left(\zeta-\frac{r}{Z}\right)}, \\
\chi_{T 3}(\psi(\tau-1)) & =\frac{\left(2-\frac{r}{Z}\right) \psi(\tau-1)}{\left(\frac{r}{Z}+2\right)} \\
& -\frac{((2+\zeta)(\eta-1)+1+2 \xi) b_{p}}{\left(\frac{r}{Z}+2\right)}, \\
\psi_{T 1} & =\frac{1}{2}\left((1+\xi)+\frac{Z}{r}\right) b_{p}, \\
\psi_{T 2} & =\frac{1}{2}\left(\eta+\xi+\frac{Z(1-\zeta(\eta-1))}{r}\right) b_{p} \quad\left(>\psi_{T 1}\right),
\end{aligned}
$$

where $D \chi_{k i}$ is the slope of $\chi_{k i}(\psi(\tau-1)), 1 \leq i \leq 3$, and $\mathcal{R}$ is the set of real numbers.

Proof in Appendix A.

Corollary 1. If $1>\frac{r}{Z}>\zeta>0$ and Nagumo's 3SPWL Resistor $(k=N)$ is $\eta>-1$ or TD 3SPWL Resistor $(k=T)$ is $\eta>1$, then $h_{k}(k=N, T): \mathcal{R} \rightarrow \mathcal{R}$ is defined by the following:

$$
\begin{aligned}
& \psi(\tau)=h_{k}(\psi(\tau-1)) \\
& =\frac{\left(1-\frac{R}{Z}\right) \chi_{k}(\psi(\tau-1))}{1+\frac{R}{Z}}+\frac{E}{1+\frac{R}{Z}}, \\
& \text { (in the case of } 1>\frac{R}{Z} \geq 0 \text { ), or } \\
& \left.=-\chi_{k}(\psi(\tau-1)) \text {, (in the case of } R \rightarrow \infty\right) \text {, } \\
& = \begin{cases}h_{k 1}(\psi(\tau-1)) & , \psi(\tau-1)<\psi_{k 1}, \\
h_{k 2}(\psi(\tau-1)) & , \psi_{k 1} \leq \psi(\tau-1) \leq \psi_{k 2} \\
h_{k 3}(\psi(\tau-1)) & , \psi_{k 2}<\psi(\tau-1) .\end{cases} \\
& h_{k i}=\frac{\left(1-\frac{R}{Z}\right) \chi_{k i}}{1+\frac{R}{Z}}+\frac{E}{1+\frac{R}{Z}}, \\
& \text { (in the case of } 1>\frac{R}{Z} \geq 0 \text { ), or } \\
& =-\chi_{k i} \text {, (in the case of } R \rightarrow \infty \text { ). }
\end{aligned}
$$

in the case where $1>R \geq 0$ : $0<D h_{k 1}<1, D h_{k 2}<-1,0<D h_{k 3}<1$ holds, where $D h_{k i}=\frac{\left(1-\frac{R}{Z}\right) D \chi_{k i}}{1+\frac{R}{Z}}$ : slope of $h_{k i}(\psi(\tau-1)), 1 \leq i \leq 3$.

The other case is where $R \rightarrow \infty:-1<D h_{k 1}<0, D h_{k 2}>1,-1<D h_{k 3}<0$ holds, where $D h_{k i}=-D \chi_{k i}$ is the - slope of $\chi_{k i}(\psi(\tau-1)), 1 \leq i \leq 3$, and $\mathcal{R}$ is the set of real numbers.

Proof in Appendix A. 
Corollary 2. If $1>\frac{r}{Z}>\zeta>0$ and Nagumo's 3SPWL Resistor $(k=N)$ is $\eta>-1$ or TD 3SPWL Resistor $(k=T)$ is $\eta>1$, then $v\left(\tau+\frac{1}{2}, \frac{1}{2}\right)$ is explicitly determined by 1-D map $g_{k}$ of $v\left(\tau-\frac{1}{2}, \frac{1}{2}\right)$ such that the following is the case.

$$
\begin{aligned}
v\left(\tau+\frac{1}{2}, \frac{1}{2}\right)= & g_{k}\left(v\left(\tau-\frac{1}{2}, \frac{1}{2}\right), \zeta, \eta, \xi\right), \\
\psi(\tau-1)= & \frac{v\left(\tau-\frac{1}{2}, \frac{1}{2}\right)+f_{k}\left(v\left(\tau-\frac{1}{2}, \frac{1}{2}\right), \zeta, \eta, \xi\right)}{2}, \\
v\left(\tau+\frac{1}{2}, \frac{1}{2}\right)= & h_{k}(\psi(\tau-1))-\frac{1+\frac{R}{Z}}{1-\frac{R}{Z}} h_{k}\left(h_{k}(\psi(\tau-1))\right) \\
+ & \frac{E}{1-\frac{R}{Z}},\left(\text { in the case of } 1>\frac{R}{Z} \geq 0\right), \text { or } \\
= & -\chi_{k}(\psi(\tau-1))+\chi_{k}\left(-\chi_{k}(\psi(\tau-1))\right), \\
& \quad \text { in the case of } R \rightarrow \infty),
\end{aligned}
$$

Remark that $i\left(\tau, \frac{1}{2}\right)=f_{k}\left(v\left(\tau, \frac{1}{2}\right), \zeta, \eta, \xi\right)$, for any $\tau$.

Proof in Appendix A.

\subsection{Global Behavior of 1-D Map}

Here, we provide Theorem 2 to guarantee that for any initial point, every orbit by the iteration of the 1-D map of $\psi(\tau)=h(\psi(\tau-1))$ ultimately penetrates an invariant interval set.

Theorem 2. If $1>\frac{r}{Z}>\zeta>0,1>\frac{R}{Z} \geq 0$ and Nagumo's 3SPWL Resistor $(k=N)$ : $\eta>-1,\left(\xi+\frac{\frac{R}{Z} \zeta-\frac{r}{Z}}{Z}\right)-\frac{E}{b_{p}}<0$, and $\left(\xi-\frac{\eta\left(\frac{R}{Z} \zeta-\frac{r}{Z}\right)}{Z}\right)-\frac{E}{b_{p}}>0$ or TD 3SPWL Resistor $(k=T)$ : $\eta>1,0>-\frac{E}{b_{p}}+\left(1+\xi+\frac{\frac{R}{Z}}{\frac{Y}{Z}}\right)+\frac{(\eta-1)\left(\frac{r}{Z}-\zeta\right)\left(1+\frac{R}{Z}\right)}{2 \frac{r}{Z}}$, and $0<-\frac{E}{b_{p}}+\left(\eta+\xi+\frac{(1-(\eta-1) \zeta) \frac{R}{Z}}{\frac{r}{Z}}\right)-$ $\frac{(\eta-1)(r-Z \zeta)\left(1+\frac{R}{Z}\right)}{2 r}$, then the following is the case.

1. The 1-D map $\psi(\tau)=h_{k}(\psi(\tau-1))$ has a unique unstable fixed point $\psi_{k *}$ in the interval $\left[\psi_{k 1}, \psi_{k 2}\right]$, where

$\psi_{N *}=\frac{(R-Z) \zeta \xi b_{p}}{2(R \zeta-r)}+\frac{E(Z \zeta-r)}{2(R \zeta-r)}$,

$\psi_{T *}=\frac{(Z-R)(\zeta(1+\xi)+1) b_{p}}{2(r-R \zeta)}+\frac{E(r-Z \zeta)}{2(r-R \zeta)}(>0)$.

2. There exists an invariant interval $I_{k \psi}=\left[h_{k}\left(\psi_{k 2}\right), h_{k}\left(\psi_{k 1}\right)\right] \supset\left[\psi_{k 1}, \psi_{k 2}\right]$ such that $h_{k}\left(I_{k \psi}\right) \subset$ $I_{k \psi}$ and $h_{k}\left(\psi_{k 2}\right)<\psi_{k 1}<\psi_{k 2}<h_{k}\left(\psi_{k 1}\right)$, where $h_{N}\left(\psi_{N 1}\right)=\frac{\left(1-\frac{R}{Z}\right)\left(\zeta+(1-\xi) \frac{r}{Z}\right) b p}{2 \frac{r}{Z}\left(1+\frac{R}{Z}\right)}+\frac{E}{1+\frac{R}{Z}}$,

$h_{N}\left(\psi_{N 2}\right)=\frac{\left(-1+\frac{R}{Z}\right)\left(\eta \zeta+(\eta+\tilde{\zeta}) \frac{r}{Z}\right) b p}{2 \frac{r}{Z}\left(1+\frac{R}{Z}\right)}+\frac{E}{1+\frac{R}{Z}}$,

$h_{T}\left(\psi_{T 1}\right)=\frac{\left(1-\frac{R}{Z}\right)\left(1-\frac{r}{Z}(1+\tau)\right) b p}{2 \frac{r}{Z}\left(1+\frac{R}{Z}\right)}+\frac{E}{1+\frac{R}{Z}}$,

$h_{T}\left(\psi_{T 2}\right)=\frac{\left(-1+\frac{R}{Z}\right)\left((\eta-1) \zeta-1+(\eta+\xi) \frac{r}{Z}\right) b p}{2 \frac{r}{Z}\left(1+\frac{R}{Z}\right)}+\frac{E}{1+\frac{R}{Z}}$.

3. For any $\psi_{0} \in(-\infty, \infty)$, there exists $j$ th iterate of $h_{k}$ such that $h_{k}^{j}\left(\psi_{0}\right) \in I_{k \psi}, j(>0) \in \mathcal{Z}$, where $h_{k}^{j}$ denotes the jth iterate of $h_{k}$, i.e., $h_{k}^{j}$ is the j-fold composition of $h_{k}$ with itself. $\mathcal{Z}$ is the set of non negative integers.

Proof in Appendix A. 


\section{Formal Chaos Existing Conditions of 1-D Maps}

As mentioned before, binary sequences based on observable chaotic behavior produced by 1-D maps are well known to have good statistical properties useful for applications relative to several digital communication systems $[4,13]$. However, the sufficient conditions for the existence of observable chaos are so strong that most 1-D maps cannot be rigorously proved to possess observable chaos. Furthermore, once the existence of formal chaos is guaranteed, the degree of the observability of the chaos can be checked by the existence of maximal positive Lyapunov exponent [14] through computer simulations from the viewpoint of engineering. Hence, we focus on the sufficient condition that formal chaos in Definition 1 exists in a 1-D map.

\subsection{Formal Chaos Existing Conditions of a General 1-D Map}

In this subsection, in order to verify formal chaos existence for 1-D map easily, we provide Theorem 3 to guarantee the formal chaos existence in Definition 1 in the case of $m=2$. Although a similar 1-D map to the map in Theorem 3 appears in Devaney or Aoki's mathematical proof process for " $\varphi$ has a homoclinic point" implying Definition 1 in [15,21], the existence of the similar 1-D map has not been considered as an easy-to-use sufficient condition implying Definition 1.

Therefore, the authors propose to adopt the existence of the similar 1-D map as the sufficient condition implying Definition 1 and to summarize this condition as Theorem 3. This theorem can be regarded as 1-D map version of Moser's theorems [14,19] for two dimensional maps.

Theorem 3. Let I be a closed interval of real numbers. Let $f$ be a continuous piecewise differentiable mapping from $I$ to itself and $D f$ be a derivative of $f$.

A1 There exist $I_{0}$ and $I_{1}$ disjoint closed subintervals in I such that $I_{0} \cup I_{1} \subset f\left(I_{0}\right)$ and $I_{0} \cup I_{1} \subset$ $f\left(I_{1}\right)$.

$A 2|D f(x)|>1$ holds for any $x$ in $I_{0} \cup I_{1}$. If $f$ satisfies $A 1$ and $A 2$, then the invariant set $\Lambda=\cap_{0 \leq n \leq \infty}(f)^{n}\left(I_{0} \cup I_{1}\right) \subset I$ exists, and the 1-D map $f$ on $\Lambda$ is topologically conjugate to the shift dynamics $\left(\sigma, \Sigma_{2}\right)$ with 2 symbols. The one-dimensional map $f$ on $\Lambda$ is mathematically chaotic.

Proof in Appendix A.

\subsection{Formal Chaos Existing Conditions of a 1-D Map Family}

As mentioned precisely in the later subsections, for a lossless transmission circuit with a dc bias voltage source in series with a load resistor at one side terminal and with a three-segment piecewise linear resistor at another side terminal, the existence of a threesegment piecewise linear 1-D map describing the dynamics of the circuit, the existence of the invariant interval of the 1-D map, and the coordinate transformation from the invariant interval to the normalized interval $I=[0,1]$ are provided.

Therefore, in this subsection, we pay our attention to the normalized 1-D map on the normalized interval $I$ after applying the coordinate transformation to the three-segment piecewise linear 1-D map. We discuss the formal chaos verification of the normalized threesegment piecewise linear 1-D map $H$ on $I$ such that the absolute slopes of the map are either greater or less than unity. In general such map $H$ satisfies Theorem 3 under very narrow range-limited circuit parameters. Instead of $H$, focusing on $H^{2}$ of which the circuit parameters are expected to have the broader range implying that $H^{2}$ satisfies Theorem 3 , we provide Theorem 5 to guarantee that the dynamics of $H^{2}$ on $I$ is topologically conjugate with respect to the shift dynamics $\left(\sigma, \Sigma_{2}\right)$ with two symbols. Since one tends to consider it as routine work for providing the sufficient inequality conditions of Theorem 5 , once again we note that,in order to obtain the inequality conditions, one needs an elaborate symbolical manipulation technique. 
We consider the normalized 1-D map as follows (see in Figure 7):

$$
x_{n+1}=H\left(x_{n}\right)= \begin{cases}H_{1}\left(x_{n}\right)=\frac{\left(1-n_{1}\right) x_{n}}{k_{1}}+n_{1} & , x_{n}<k_{1}, \\ H_{2}\left(x_{n}\right)=\frac{-x_{n}+k_{1}}{k_{2}-k_{1}}+1 & , k_{1} \leq x_{n} \leq k_{2}, \\ H_{3}\left(x_{n}\right)=\frac{n_{2}\left(x_{n}-k_{2}\right)}{1-k_{2}} & , k_{2}<x_{n},\end{cases}
$$

where $I=[0,1] \subset \mathcal{R}, x_{n}, x_{n+1} \in I$, and $\mathcal{R}$ is the set of real numbers.

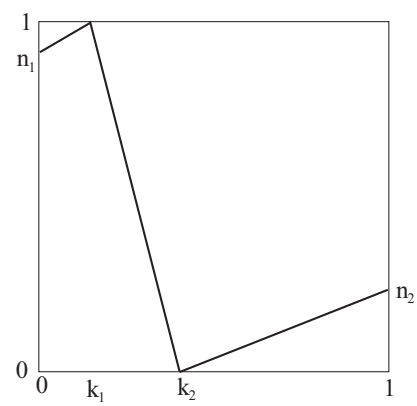

Figure 7. Normalized 1-D map.

Theorem 4. If $1-k_{1}\left(k_{2}-k_{1}\right)>n_{1}>n_{2}>\left(k_{2}-k_{1}\right)\left(1-k_{2}\right), n_{1}>1-n_{2}>k_{2}>n_{2}>$ $k_{1}>1-n_{1}$ and $0<k_{1}, k_{2}, n_{1}, n_{2}<1$, then the following is the case:

$$
\begin{aligned}
& x_{n+1}=H^{2}\left(x_{n}\right)=\left\{\begin{array}{l}
H_{1}\left(H_{1}\left(x_{n}\right)\right), \text { when } x_{n}<-\frac{\left(k_{1}-n_{1}\right) k_{1},}{n_{1}-1}, \\
H_{2}\left(H_{1}\left(x_{n}\right)\right) \text {, when }-\frac{\left(k_{1}-n_{1}\right) k_{1} \leq x_{n}}{n_{1}-1} \leq-\frac{\left(k_{2}-n_{1}\right) k_{1}}{n_{1}-1}, \\
H_{3}\left(H_{1}\left(x_{n}\right)\right), \text { when }-\frac{\left(k_{2}-n_{1}\right) k_{1}}{n_{1}-1} \leq x_{n}<k_{1}, \\
H_{3}\left(H_{2}\left(x_{n}\right)\right), \text { when } k_{1} \leq x_{n}<k_{2}-k_{2}\left(k_{2}-k_{1}\right), \\
H_{2}\left(H_{2}\left(x_{n}\right)\right) \text {, when } k_{2}-k_{2}\left(k_{2}-k_{1}\right) \leq x_{n}<k_{2}\left(1-k_{1}\right)+k_{1}^{2}, \\
H_{1}\left(H_{2}\left(x_{n}\right)\right), \text { when } k_{2}\left(1-k_{1}\right)+k_{1}^{2} \leq x_{n}<k_{2}, \\
H_{1}\left(H_{3}\left(x_{n}\right)\right), \text { when } k_{2} \leq x_{n}<k_{2}+\frac{k_{1}\left(1-k_{2}\right)}{n_{2}}, \\
H_{2}\left(H_{3}\left(x_{n}\right)\right), \text { when } k_{2}+\frac{k_{1}\left(1-k_{2}\right)}{n_{2}} \leq x_{n}<k_{2}+\frac{k_{2}\left(1-k_{2}\right)}{n_{2}}, \\
H_{3}\left(H_{3}\left(x_{n}\right)\right), \text { when } k_{2}+\frac{k_{2}\left(1-k_{2}\right)}{n_{2}}<x_{n},
\end{array}\right. \\
& -\frac{\left(k_{1}-n_{1}\right) k_{1}}{n_{1}-1}<-\frac{\left(k_{2}-n_{1}\right) k_{1}}{n_{1}-1}<0, \\
& 0<k_{1}<k_{2}-k_{2}\left(k_{2}-k_{1}\right) \text {, } \\
& k_{2}-k_{2}\left(k_{2}-k_{1}\right)<k_{2}\left(1-k_{1}\right)+k_{1}^{2}<k_{2} \text {, } \\
& k_{2}<k_{2}+\frac{k_{1}\left(1-k_{2}\right)}{n_{2}}<1 \text {, } \\
& 1<k_{2}+\frac{k_{2}\left(1-k_{2}\right)}{n_{2}},
\end{aligned}
$$

such that $\left|D H_{1} H_{1}\right|=\left|\frac{\left(1-n_{1}\right)^{2}}{k_{1}^{2}}\right|<1,\left|D H_{2} H_{2}\right|=\left|\frac{1}{\left(k_{2}-k_{1}\right)^{2}}\right|>1$, $\left|D H_{3} H_{3}\right|=\left|\frac{n_{2}^{2}}{\left(1-k_{2}\right)^{2}}\right|<1,\left|D H_{3} H_{1}\right|=\left|D H_{1} H_{3}\right|=\left|\frac{n_{2}\left(1-n_{1}\right)}{k_{1}\left(1-k_{2}\right)}\right|<1$, $\left|D H_{3} H_{2}\right|=\left|D H_{2} H_{3}\right|=\left|\frac{-n_{2}}{\left(k_{2}-k_{1}\right)\left(1-k_{2}\right)}\right|>1,\left|D H_{1} H_{2}\right|=\left|D H_{2} H_{1}\right|=\left|\frac{-\left(1-n_{1}\right)}{k_{1}\left(k_{2}-k_{1}\right)}\right|>1$, where $D H_{i} H_{j}$ is the slope of $H_{i}\left(H_{j}\left(x_{n}\right)\right), 1 \leq i, j \leq 3$. 
Proof in Appendix A.

Theorem 5. If $1-k_{1}\left(k_{2}-k_{1}\right)>n_{1}>n_{2}>\left(k_{2}-k_{1}\right)\left(1-k_{2}\right), n_{1}>1-n_{2}>k_{2}>n_{2}>$ $k_{1}>1-n_{1}, k_{2}-k_{2}\left(k_{2}-k_{1}\right)>\frac{k_{2}-n_{2}}{k_{2}-k_{1}}$ and $0<k_{1}, k_{2}, n_{1}, n_{2}<1$, then the following is the case:

1. There exist disjoint closed intervals $I_{0}=\left[k_{2}-k_{2}\left(k_{2}-k_{1}\right), k_{2}\left(1-k_{2}\right)+k_{1}^{2}\right] \subset I$ and $I_{1}=\left[k_{2}+\frac{k_{1}\left(1-k_{2}\right)}{n_{2}}, 1\right] \subset I$ such that $I_{0} \cup I_{1} \subset H^{2}\left(I_{0}\right)=H_{2} H_{2}\left(I_{0}\right), I_{0} \cup I_{1} \subset H^{2}\left(I_{1}\right)=$ $H_{2} H_{3}\left(I_{1}\right)$, and $\left|D H^{2}(\Psi)\right|>1$ holds for $x_{n} \in I_{0} \cup I_{1}$, where $D H^{2}\left(x_{n}\right)$ is the slope of $H^{2}\left(x_{n}\right)$.

2. Invariant set $\Lambda=\cap_{0 \leq n \leq \infty}\left(H^{2}\right)^{n}\left(I_{0} \cup I_{1}\right) \subset$ I exists, and $H^{2}$ on $\Lambda$ is topologically conjugate to the shift dynamics $\left(\sigma, \Sigma_{2}\right)$ with two symbols. $H^{2}$ on $\Lambda$ is mathematically chaotic.

Proof in Appendix A.

\subsection{Formal Chaos Existing Conditions of 1-D Maps of Lossless Transmission Circuits}

Now, we discuss the method for verifying formal chaos in the dynamics of the 1-D map of $\psi(\tau)=h_{k}(\psi(\tau-1)),(k=N, T)$ rigorously. In the following, we assume that the conditions of Theorem 2 are satisfied. Then, $\psi(\tau)=h_{k}(\psi(\tau-1))$ has a unique unstable fixed point $\psi_{* k} \in I_{\psi k}$ and an invariant interval $I_{\psi k}$ such that $h\left(I_{\psi k}\right) \subset I_{\psi k}$, and for any $\psi_{0} \in(-\infty, \infty)$, there exists $j$ th iterate of $h_{k}$ such that $h_{k}^{j}\left(\psi_{0}\right) \in I_{\psi k}, j(>0) \in \mathcal{Z}$. Thus, eventually all we have to consider are the dynamics of $\psi(\tau)=h_{k}(\psi(\tau-1))$ on the invariant interval of $I_{\psi k}$. To this end, we consider the following coordinate transformation and maps. Using Equations (26) and (27), we provide Theorem 4. By further using Theorem 3, we provide Theorem 5 in order to guarantee that the dynamics of $h_{k}^{2}$ on an invariant subset $\subset I_{\psi k}$ is topologically conjugate to the shift dynamics $\left(\sigma, \Sigma_{2}\right)$ with two symbols.

When the conditions of Theorem 2 are satisfied, the coordinate transformation $p_{k}$ $(\mathrm{k}=\mathrm{N}, \mathrm{T}): I_{k \psi} \rightarrow I$ (or $p_{k}^{-1}: I \rightarrow I_{k \psi}$ ) is defined as follows:

$$
\begin{aligned}
\Psi & =p_{k}(\psi)=\frac{\psi-h_{k}\left(\psi_{k 2}\right)}{h_{k}\left(\psi_{k 1}\right)-h_{k}\left(\psi_{k 2}\right)} \\
p_{k}^{-1}(\Psi) & =\left(h_{k}\left(\psi_{k 1}\right)-h_{k}\left(\psi_{k 2}\right)\right) \Psi+h_{k}\left(\psi_{k 2}\right) .
\end{aligned}
$$

where $I_{k \psi}=\left[h\left(\psi_{k 2}\right), h\left(\psi_{k 1}\right)\right], I=[0,1]$.

\section{Formal Chaos Existence and Bifurcation Behavior of 1-D Maps by Using Maple}

In this section, we pay our attention to the 1-D maps derived from the transmission line circuits with TD 3SPWL Resistor $F_{T}$ at another side terminal because the characteristics of TD 3SPWL Resistor $F_{T}$ is more general than the characteristics of Nagumo's 3SPWL resistor $F_{N}$. Then, we present an example of formal chaos existence and several examples of bifurcation behavior of 1-D maps. Using the degree of observability of chaotic states in terms of Lyapunov exponent, we show the observability of formal chaos in such bifurcation processes.

\subsection{An Example of Formal Chaos Existence}

In this subsection, using Maple, we present an example of the existence of formal chaos. Under the condition such that $V_{\max }=10[\mathrm{~V}], Z=300[\Omega], b_{p}=\frac{3}{50}, r=\frac{3}{25}, \eta=\frac{20}{3}, \zeta=\frac{1}{10}$, $E=\frac{143}{320}$ and $R=\frac{7}{16}$, the following is the case.

1. $\quad \eta>1,1>r>\zeta>0$, and $1>R>0$ hold;

2. $E=\frac{143}{320}>b_{p}\left(1+\frac{R}{r}\right)+b_{p} \frac{(\eta-1)(r-\zeta)(1+R)}{2 r}=\frac{3067}{9600}$, and $E=\frac{143}{320}<b_{p}\left(\eta+\frac{(1-(\eta-1) \zeta) R}{r}\right)-$ $b_{p} \frac{(\eta-1)(r-\zeta)(1+R)}{2 r}=\frac{1453}{3200}$ hold;

3. $k_{1}=\frac{23}{561}, k_{2}=\frac{460}{1683}, n_{1}=\frac{235}{238}$, and $n_{2}=\frac{57,481}{227,953}$ hold;

4. $0<k_{1}, k_{2}, n_{1}, n_{2}<1$ holds; 
5. $\quad n_{1}=\frac{235}{238}>1-n_{2}=\frac{170,472}{227,953}>k_{2}=\frac{460}{1683}>n_{2}=\frac{57,481}{227,953}>k_{1}=\frac{23}{561}>1-n_{1}=\frac{3}{238}$ holds;

6. $\quad 1-k_{1}\left(k_{2}-k_{1}\right)=\frac{55,010}{55,539}>n_{1}=\frac{235}{238}>n_{2}=\frac{57,481}{227,953}>\left(k_{2}-k_{1}\right)\left(1-k_{2}\right)=\frac{28,129}{166,617}$ holds.

7. $k_{2}-k_{2}\left(k_{2}-k_{1}\right)=\frac{34,960}{166,617}>\frac{k_{2}-n_{2}}{k_{2}-k_{1}}=\frac{43,411}{476,629}$ holds.

Hence, all the conditions of Theorems 1, 2, 4, and 5 and Corollaries 1 and 2 are satisfied. $H^{2}(\Psi)$ has obviously the following properties:

1. There exist disjoint intervals $I_{0}=\left[\frac{34960}{166617}, \frac{14651}{55539}\right] \subset I$ and $I_{1}=\left[\frac{92897}{237303}, 1\right] \subset I$ such that $I_{0} \cup I_{1} \subset H^{2}\left(I_{0}\right)=H_{2} H_{2}\left(I_{0}\right), I_{0} \cup I_{1} \subset H^{2}\left(I_{1}\right)=H_{2} H_{3}\left(I_{1}\right)$, and $\left|D H^{2}(\Psi)\right|>1$ holds for $\Psi \in I_{0} \cup I_{1}$, where $D H^{2}(\Psi)$ is the slope of $H^{2}(\Psi)$;

2. Invariant set $\Lambda=\bigcap_{0 \leq n \leq \infty}\left(H^{2}\right)^{n}\left(I_{0} \cup I_{1}\right) \subset I$ exists, and $H^{2}$ on $\Lambda$ is topologically conjugate to the shift dynamics $\left(\sigma, \Sigma_{2}\right)$ with two symbols. $H^{2}$ on $\Lambda$ is mathematically chaotic.

In the following, we show $f(v, \zeta, \eta), H(\Psi)$, and $H^{2}(\Psi)$ and illustrate the $v$ - $i$ characteristic of $f(v, \zeta, \eta)$ and the graphs of $H(\Psi)$ and $H^{2}(\Psi)$ in Figures 8 and 9 , respectively. Further graphical iterative paths of $H^{2}(\Psi)$ and voltage map by Corollary 2 and iterative time series data of the voltage map at iterative time $n$ are illustrated in Figures 10-12, respectively. The conditions of these graphical iterations are as follows: the initial point of these iterations is $x_{0}=\frac{52,828}{227,953} \subset I_{0}$, and with the use of Maple 8, the graphical iterations in these figures are illustrated by 2000 iterations such that the iterations from first until 1000 are not used in the total 3000 iterations. In addition, the real parameters of the conditions above are reasonable because $E_{d}=\frac{3}{5}[\mathrm{~V}], E_{p}=4[\mathrm{~V}], R_{1}=R_{2}=36[\Omega], R_{4}=\frac{12,960}{11 R_{3}}[\Omega]$, $R_{4}=\frac{360}{11}[\Omega]$, if $R_{3}=36[\Omega], E_{B}=\frac{143}{32}[\mathrm{~V}]$, and $R_{L}=\frac{525}{4}[\Omega]$. The real transmission line circuit with three-segment piecewise linear resistor can be implemented.

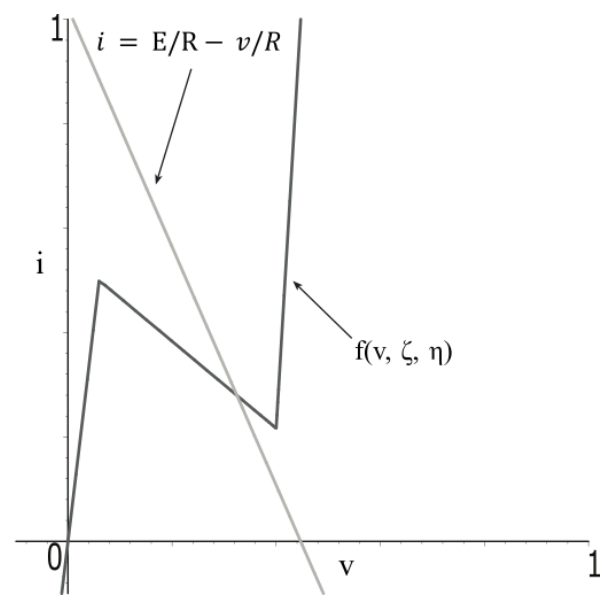

Figure 8. $v-i$ characteristic of $f(v)$.

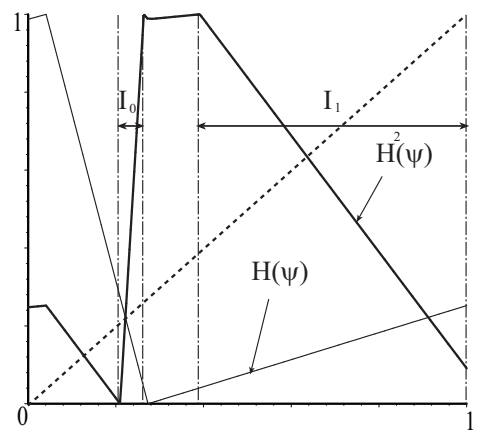

Figure 9. $H(\Psi)$ and $H^{2}(\Psi)$. 


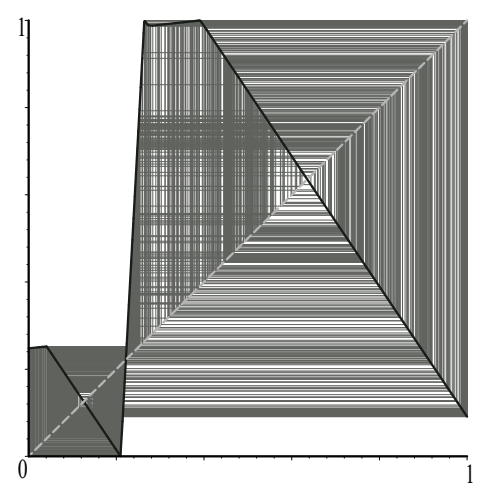

Figure 10. Iterative paths of $H^{2}(\Psi)$.

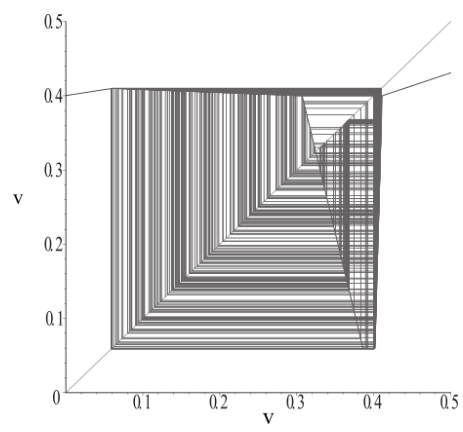

Figure 11. Iterative paths of voltage map.

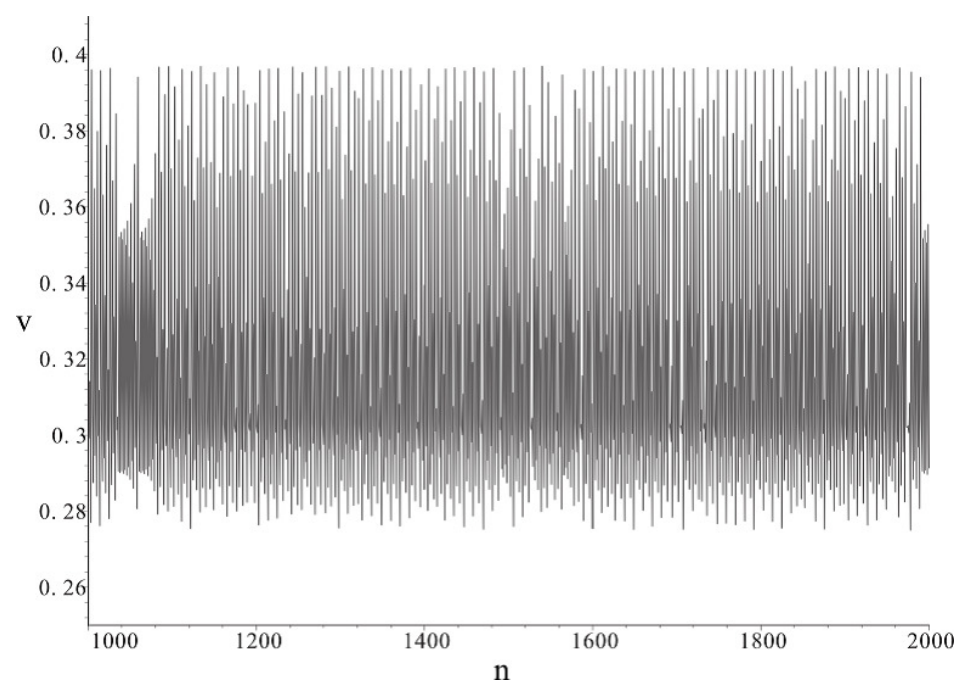

Figure 12. Time series data of voltage map at iterative time $n$.

\subsection{Several Examples of Bifurcation Behavior of 1-D Maps}

In this subsection, by using Maple 8, we present various bifurcation behavior (including the found formal chaos) of 1-D voltage maps of Equation (18) with maximal Lyapunov exponent. The degree of observability of chaotic states is given by Lyapunov exponent $\mu\left(v_{0}\right)$ of Equation (28) for any initial point $v_{0}$ [14].

$$
\mu\left(v_{0}\right)=\frac{1}{N} \sum_{i=0}^{N-1}\left|\frac{d g_{k}}{d v}\left(v_{i}, \zeta, \eta, \xi\right)\right|
$$

Here, codimension one bifurcation diagrams with one of the bifurcation parameters: $\zeta, \eta, \xi$, or $E$, and representative iterative paths are summarized as follows. With one of 
the following bifurcation parameters from (1) bifurcation parameter $\zeta$ until (4) bifurcation parameter $E$, the codimension one bifurcation diagrams including the found formal chaos togethar with the Lyapunov exponents, are illustrated in Figures 13-16, respectively $\left(V_{\max }=10[\mathrm{~V}], I_{\max }=\frac{1}{30}[\mathrm{~A}], R_{\max }=300[\Omega]\right)$.

(1) bifurcation parameter $\zeta: \eta=\frac{20}{3}, \xi=0, Z=1, E=\frac{143}{320}, \frac{R}{Z}=\frac{7}{16}$.

(2) bifurcation parameter $\eta: \zeta=\frac{1}{10}, \xi=0, Z=1, E=\frac{143}{320}, \frac{R}{Z}=\frac{7}{16}$.

(3) bifurcation parameter $\zeta: \zeta=\frac{1}{10}, \eta=\frac{20}{3}, Z=1, E=\frac{143}{320}, \frac{R}{Z}=\frac{7}{16}$.

(4) bifurcation parameter $E: \zeta=\frac{1}{10}, \eta=\frac{20}{3}, \xi=0, Z=1, \frac{R}{Z}=\frac{7}{16}$.

Representative iterative paths in bifurcation diagrams of Figures 13-16 are illustrated in Figures 17-20, respectively. Graphical iterations in these figures are illustrated by 60 iterations such that the iterations from first until 40 are not used in the total 100 iterations. Since the voltage maps are composite maps consisting of incident waves or reflected waves, etc., it takes too much computation time to obtain bifurcation diagrams with Lyapunov exponents in Figures 13-16. Therefore, the number of iterations for the voltage maps is intentionally reduced.

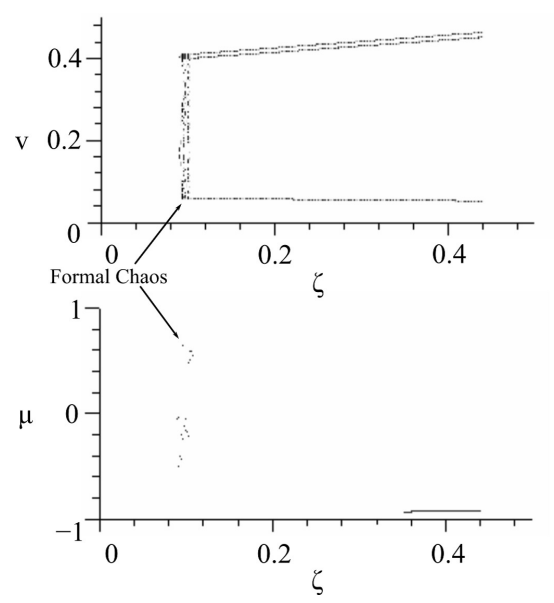

Figure 13. Bifurcation diagram with $\zeta$ parameter.

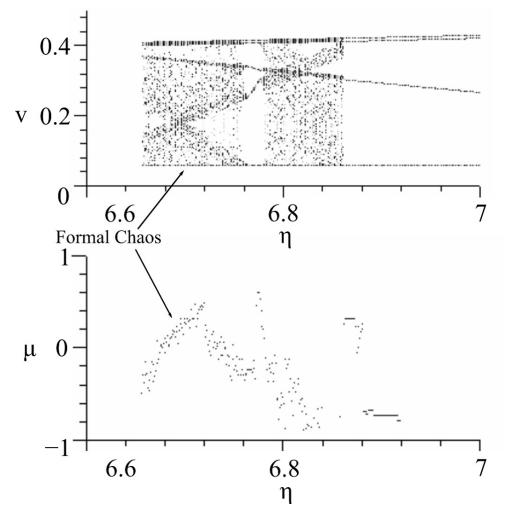

Figure 14. Bifurcation diagram with $\eta$ parameter. 


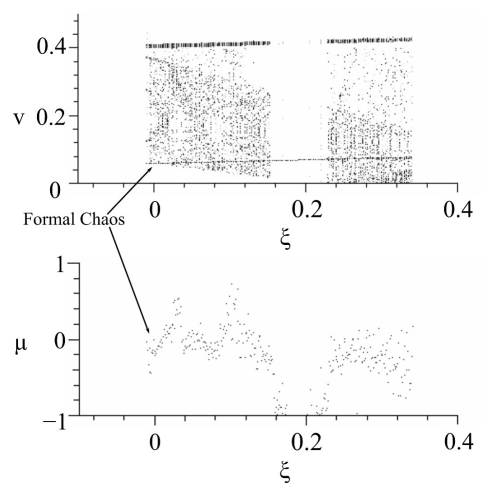

Figure 15. Bifurcation diagram with $\xi$ parameter.
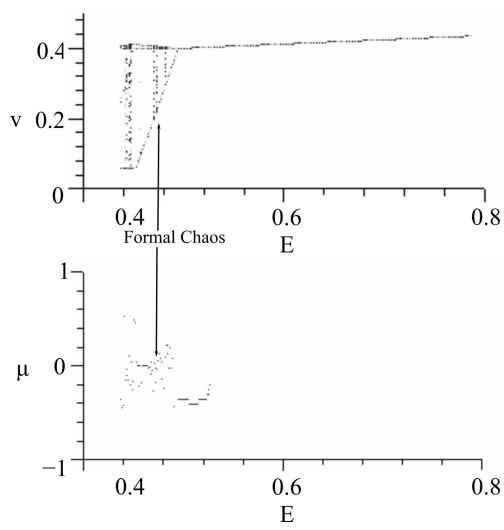

Figure 16. Bifurcation diagram with $E$ parameter.

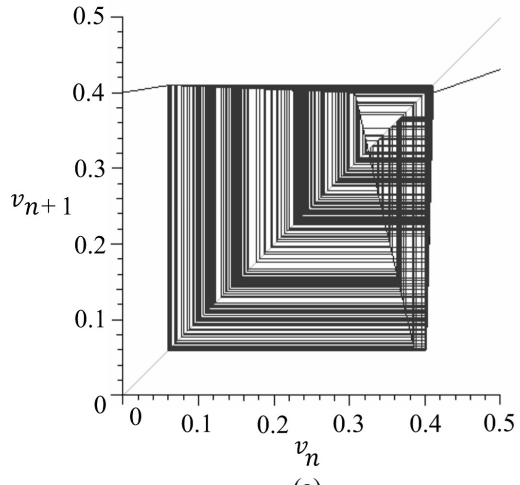

(a)

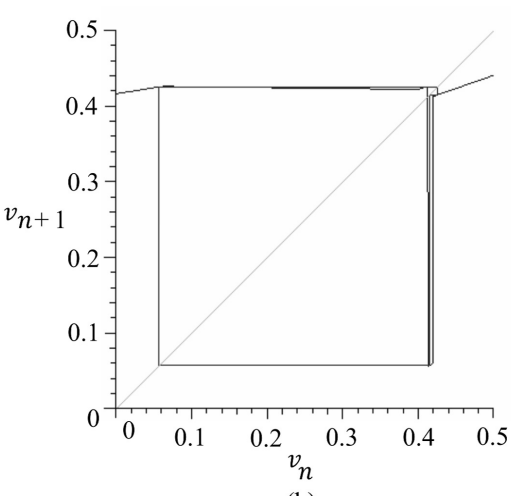

(b)

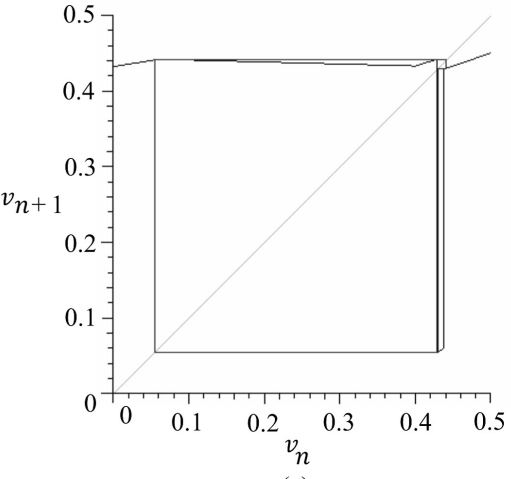

(c)

Figure 17. Iterative paths with the initial point: $v_{0}=0.5$. (a) $\zeta=0.1$. (b) $\zeta=0.2$. (c) $\zeta=0.3$.

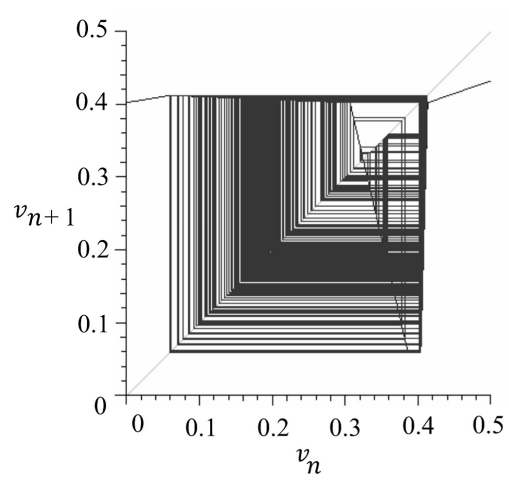

(a)

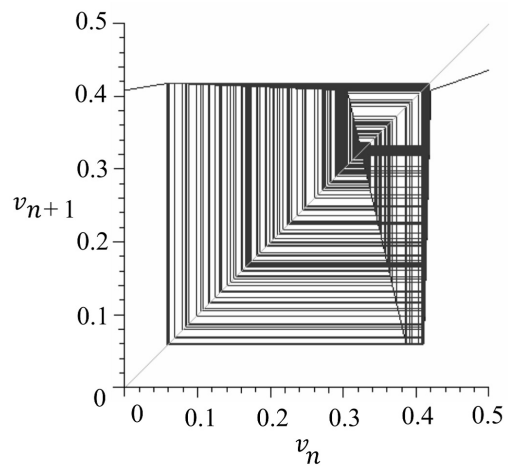

(b)

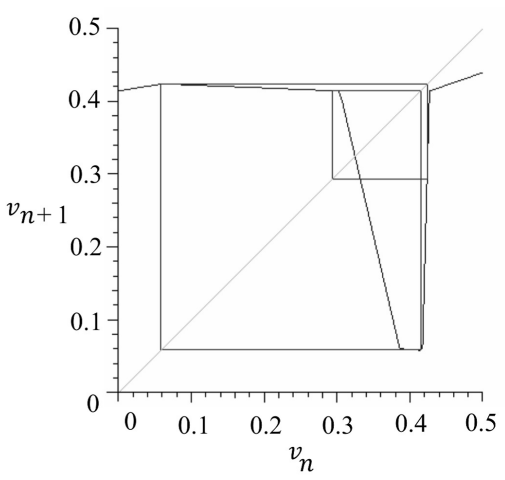

(c)

Figure 18. Iterative paths with the initial point: $v_{0}=0.5$. (a) $\eta=6.7$. (b) $\eta=6.8$. (c) $\eta=6.9$. 


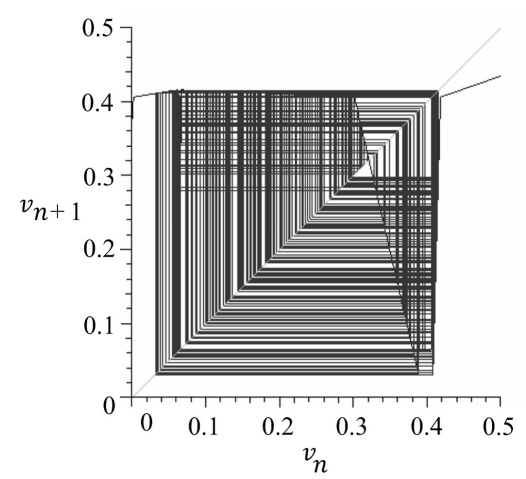

(a)

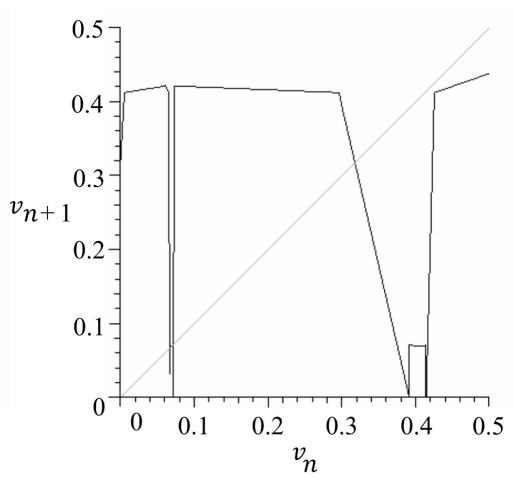

(b)

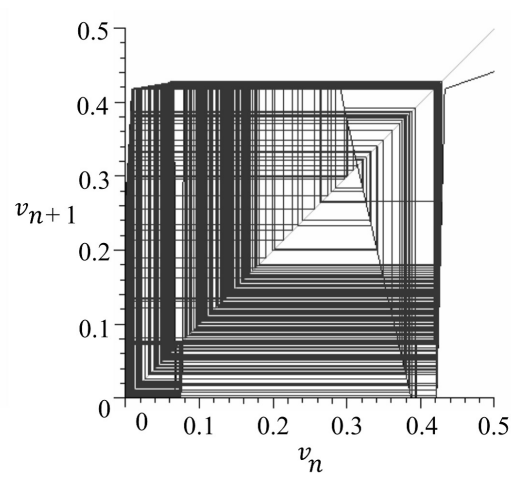

(c)

Figure 19. Iterative paths with the initial point: $v_{0}=0.5$. (a) $\xi=0.1$. (b) $\xi=0.2$. (c) $\xi=0.3$.

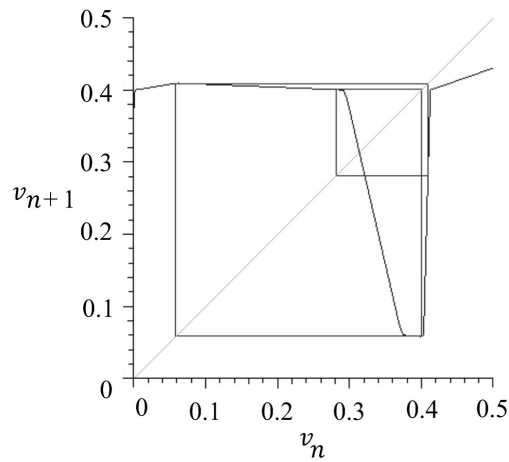

(a)

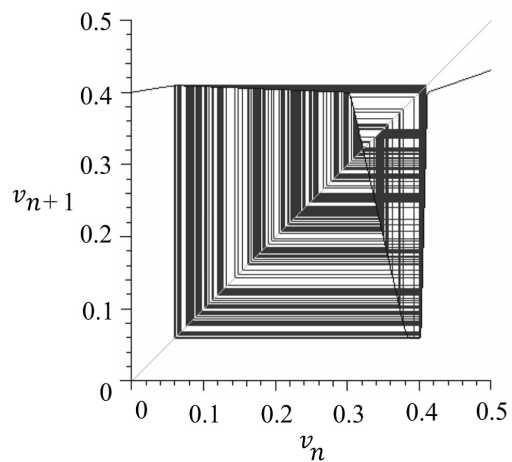

(b)

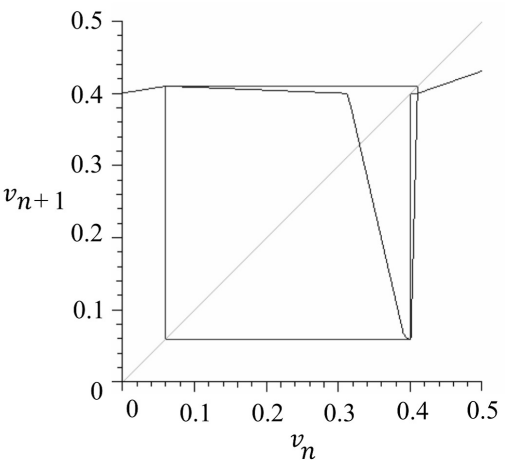

(c)

Figure 20. Iterative paths with the initial point: $v_{0}=0.5$. (a) $E=0.44$. (b) $E=0.445$. (c) $E=0.45$.

\section{Conclusions}

1. We have described an implicit 1-D map of the incident and reflected waves that is derived from a lossless transmission line circuit with a dc bias voltage source in series with a load resistor at one side terminal and with a three-segment piecewise linear resistor at another side terminal.

2. We have provided Theorem 1 establishing a 1-D map such as an incident wave: $\psi(\tau-1) \rightarrow$ a reflected wave: $\phi(\tau)$; Corollary 1 establishing 1-D map such as an incident wave: $\psi(\tau-1) \rightarrow$ a reflected wave: $\phi(\tau)$; and Corollary 2 establishing 1-D map such as a voltage: $v\left(\tau-\frac{1}{2}, \frac{1}{2}\right) \rightarrow$ a voltage: $v\left(\tau+\frac{1}{2}, \frac{1}{2}\right)$ in the case of Nagumo's 3SPWL resistor or TD 3SPWL Resistor at the side terminal: $x=\frac{1}{2}$.

3. We have provided Theorem 3 such as an easy-to-use sufficient condition implying formal chaos existence in Definition 1.

4. We have provided Theorem 2 to guarantee that for any initial point, every orbit by the iteration of the 1-D map ultimately penetrates in an invariant interval.

5. We have provided Theorems 2 and 5 to guarantee that the dynamics of the second iterate map of the 1-D map on an invariant subset of the invariant interval has formal chaos.

6. We have found that formal chaos exists under the condition such that $V_{\max }=10$ [V], $Z=300[\Omega], b_{p}=\frac{3}{50}, r=\frac{3}{25}, \eta=\frac{20}{3}, \zeta=\frac{1}{10}, E=\frac{143}{320}$, and $R=\frac{7}{16}$.

7. We have obtained the codimension of one type of four various bifurcation diagrams including the found formal chaos, with one of the bifurcation parameters: $\zeta, \eta, \xi$, or $E$. From each bifurcation diagram with the Lyapunov exponent, the obsavability of the found formal chaos is considered to be rather high.

8. As with the case of [12] such that the hidden dynamics of the circuit based on the integrated device has been unveiled on the basis of an analogy with the well-known Colpitts oscillator with the chaotic oscillations, we think that the hidden dynamics of imperfect transmission lines with parasitic effects and nonidealities inside real 
integrated devices can be unveiled on the basis of an analogy with the transmission line circuit of Equations (2)-(6) with formal chaos and that the parameters of the hidden dynamics can also be estimated by synchronizing a transmission line circuit with the chaotic oscillations acquired from the experimental circuit.

9. We will report bifurcation processes with each of bifurcation parameters, $Z$ or $R$, because nonlinear phenomena such as intermittency of chaos or blue sky bifurcation are observed in simulations.

10. We will establish a method to find the bifurcation parameter ranges such that formal chaos exists for applications relative to several digital communication systems.

Author Contributions: H.O. supervised this research. H.N. and H.O. initiated the research, and contributed of a lossless transmission line circuit conceptualization and investigation of the transmission line circuit. K.O. and H.O. managed all of the research. H.O. provided theorems, and proofs. H.O. and K.O. contributed development of MAPLE simulation algorithm. K.O. also carried out the simulations. K.I. contributed validation of the methodology. K.O., K.I. and H.O. contributed the all of analysis result. The original manuscript was written by K.O., K.I. and H.O. And all of authors reviewed and edited the manuscript. All authors have read and agreed to the published version of the manuscript.

Funding: This research was supported by the research budget of the Graduate School of Electrical and Computer Engineering, Shonan Institute of Technology.

Institutional Review Board Statement: Not Applicable.

Informed Consent Statement: Not Applicable.

Data Availability Statement: Data is contained within the article.

Conflicts of Interest: The authors declare no conflict of interest.

\section{Appendix A. Proofs of Theorems and Corollaries}

Proof of Theorem 1. Each of the proof for the case $k=N$, or $T$, is given as follows.

1. In the case of $k=N$ : By using Equations (5) and (8), the break points (- $(1-$ $\left.\xi) b_{p}, f_{N}\left(-(1-\xi) b_{p}, \zeta, \eta, \xi\right)\right)$ and $\left((\eta+\xi) b_{p}, f_{N}\left((\eta+\xi) b_{p}, \zeta, \eta, \xi\right)\right)$ are transformed into $\left(\psi_{N 1}, \phi_{N 1}\right)$ and $\left(\psi_{N 2}, \phi_{N 2}\right)$, respectively, such that the following is the case.

$$
\begin{aligned}
& \psi_{N 1}=\frac{1}{2}\left(-(1-\xi)+\frac{\zeta}{r}\right) b_{p}, \phi_{N 1}=\frac{1}{2}\left((1-\xi)+\frac{\zeta}{r}\right) b_{p}, \\
& \psi_{N 2}=\frac{1}{2}\left(\eta+\xi-\frac{\zeta \eta}{r}\right) b_{p}, \phi_{N 2}=\frac{1}{2}\left(-(\eta+\xi)-\frac{\zeta \eta}{r}\right) b_{p} .
\end{aligned}
$$

$\psi_{N 2}>\psi_{N 1}$ and $\phi_{N 1}>\phi_{N 2}$ hold because $\psi_{N 2}-\psi_{N 1}=\frac{b_{p}(\eta+1)(r-\zeta)}{2 r}>0$ and $\phi_{N 1}-\phi_{N 2}=\frac{b_{p}(\eta+1)(r+\zeta)}{2 r}>0$, respectively. Since $(\psi, \phi)$-coordinate system is identical with the $-\pi / 4$ rotation of $(v, i)$-coordinate system and $\psi_{N 2}>\psi_{N 1}$ and $\phi_{N 1}>\phi_{N 2}$, as shown in Figure A1, $\phi(\tau)$ is explicitly described by a three-segment piecewise linear function of Equation (16). $0<1-r<1,1<1+r<2,0<r-\zeta<r$, and $r+\zeta<2 r$ hold because of the conditions such that $1>r>\zeta>0$ and $\eta>1$. Therefore, $0<D \chi_{N 1}=\frac{(1-r)}{(r+1)}<1,-1>D \chi_{N 2}=\frac{(\zeta+r)}{(\zeta-r)}$, and $0<D \chi_{N 3}=$ $\frac{(1-r)}{(r+1)}<1$ hold 
2. In the case of $k=T$ : By using Equations (6) and (8), the break points $\left((1+\xi) b_{p}, f_{T}((1+\right.$ $\left.\left.\xi) b_{p}, \zeta, \eta, \xi\right)\right)$ and $\left((\eta+\xi) b_{p}, f_{T}\left((\eta+\xi) b_{p}, \zeta, \eta, \xi\right)\right)$ are transformed into $\left(\psi_{T 1}, \phi_{T 1}\right)$ and $\left(\psi_{T 2}, \phi_{T 2}\right)$, respectively, such that the following is the case:

$$
\begin{aligned}
\psi_{T 1} & =\frac{1}{2}\left(1+\xi+\frac{1}{r}\right) b_{p}, \phi_{T 1}=\frac{1}{2}\left(-1-\xi+\frac{1}{r}\right) b_{p}, \\
\psi_{T 2} & =\frac{1}{2}\left(\eta+\xi+\frac{1-\zeta(\eta-1)}{r}\right) b_{p}, \\
\phi_{T 2} & =\frac{1}{2}\left(-\eta-\xi+\frac{1-\zeta(\eta-1)}{r}\right) b_{p} .
\end{aligned}
$$

$\psi_{T 2}>\psi_{T 1}$ and $\phi_{T 1}>\phi_{T 2}$ hold because $\psi_{T 2}-\psi_{T 1}=\frac{b_{p}(\eta-1)(r-\zeta)}{2 r}>0$ and $\phi_{T 1}-\phi_{T 2}=\frac{b_{p}(\eta-1)(r+\zeta)}{2 r}>0$, respectively. Since the $(\psi, \phi)$-coordinate system is identical with the $-\pi / 4$ rotation of $(v, i)$-coordinate system and $\psi_{T 2}>\psi_{T 1}$ and $\phi_{T 1}>\phi_{T 2}$, as shown in Figure A2, $\phi(\tau)$ is explicitly described by a three-segment piecewise linear function of Equation (16). $0<1-r<1,1<1+r<2,0<r-\zeta<r$, $r+\zeta<2 r, 1<2-r<2$, and $2<r+2<3$ hold because of the conditions such that $1>r>\zeta>0$ and $\eta>1$. Therefore, $0<D \chi_{T 1}=\frac{(1-r)}{(r+1)}<1,-1>D \chi_{T 2}=\frac{(\zeta+r)}{(\zeta-r)}$, and $0<D \chi_{\mathrm{T3}}=\frac{(2-r)}{(r+2)}<1$ hold.

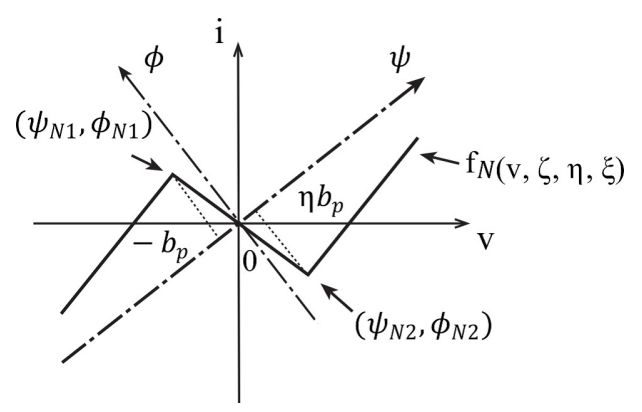

Figure A1. The characteristics of Nagumo's 3SPWL Resistor in $(\psi, \phi)$ and $(v, i)$ coordinate systems.

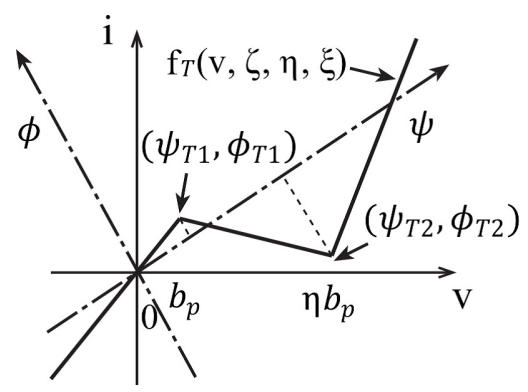

Figure A2. The characteristics of TD 3SPWL Resistor in $(\psi, \phi)$ and $(v, i)$ coordinate systems.

The proof is now complete.

Proof of Corollary 1. With the substitution of Equations (16) into (9), $\psi(\tau)=h_{k}(\psi(\tau-1)$ ), that is, Equation (17) is easily obtained. Then, $0<1-R<1$ and $1<1+R<2$, that is, $0<\frac{1-R}{1+R}<1$ hold because $1>R>0$. Therefore, under the conditions of Corollary 1 , $0<D h_{k 1}=\frac{(1-R) D \chi_{k 1}}{1+R}<1,-1>D h_{k 2}=\frac{(1-R) D \chi_{k 2}}{1+R}$, and $0<D h_{k 3}=\frac{(1-R) D \chi_{k 3}}{1+R}<1$ hold.

Proof of Corollary 2. The 1-D map in Corollary 2 is easily derived from Equations (7), (8), and (10); Corollary 1; and Theorem 1. 
Proof of Theorem 2. Under the conditions that Theorem 2 holds, the following is the case. In the case of $k=T$ : It is easy to check the following properties: (1) $\psi_{T *}>0$ holds because $r-\zeta>0$ and $r-R \zeta>0$. (2) $\psi_{T *}-\psi_{T 1}=\frac{b_{p}(\zeta-r)(R+r(1+\xi))}{2 r(r-R \zeta)}+\frac{E(r-\zeta)}{2(r-R \zeta)}>0$ and $\psi_{T 2}-\psi_{T *}=-\frac{(\zeta-r)((\eta+\xi) r+(1+(1-\eta) \zeta) R) b_{p}}{2 r(r-R \zeta)}-\frac{E(r-\zeta)}{2(r-R \zeta)}>0$, i.e., $\psi_{T *} \in\left[\psi_{T 1}, \psi_{T 2}\right]$ hold in the case of $E>b_{p}\left(1+\xi+\frac{R}{r}\right)$ and $E<b_{p}\left(\eta+\xi+\frac{(1-(\eta-1) \zeta) R}{r}\right)$.

Therefore, in the case of $E>b_{p}\left(1+\xi+\frac{R}{r}\right)+b_{p} \frac{(\eta-1)(r-\zeta)(1+R)}{2 r}$ and $E<b_{p}(\eta+\xi+$ $\left.\frac{(1-(\eta-1) \zeta) R}{r}\right)-b_{p} \frac{(\eta-1)(r-\zeta)(1+R)}{2 r}, \quad \psi_{T *} \in\left[\psi_{T 1}, \psi_{T 2}\right]$ obviously holds because $b_{p} \frac{(\eta-1)(r-\zeta)(1+R)}{2 r}>0$. Furthermore, for all $x$ such as $x<\psi_{T 1},\left(D h_{T 1}-1\right)\left(x-\psi_{T 1}\right)>0$ holds because $\left(D h_{T 1}-1\right)<0$ and $\left(x-\psi_{T 1}\right)<0$ for all $x$ such as $x<\psi_{T 1} \cdot \frac{E}{1+R}+\left(D h_{T 1}-\right.$ 1) $\psi_{T 1}=\frac{b_{p}(r-\zeta)(\eta-1)}{2 r}>0$ holds in the case of $E>b_{p}\left(1+\xi+\frac{R}{r}\right)+b_{p} \frac{(\eta-1)(r-\zeta)(1+R)}{2 r}$.

Therefore, for all $x$ such as $x<\psi_{T 1}$, the following is the case.

$$
\begin{aligned}
h_{T}(x)-x & =\left(D h_{T 1}-1\right)\left(x-\psi_{T 1}\right)+\frac{E}{1+R} \\
& +\left(D h_{T 1}-1\right) \psi_{T 1}>0 .
\end{aligned}
$$

In a similar manner as the above, for all $x$ such as $x>\psi_{T 2},\left(1-D h_{T 3}\right)\left(x-\psi_{T 2}\right)>0$ holds because $\left(1-D h_{T 3}\right)>0$ and $\left(x-\psi_{T 2}\right)>0$ for all $x$ such as $x>\psi_{T 2}$.

$-\frac{E}{1+R}+\left(1-D h_{T 3}\right) \psi_{T 2}=\frac{b_{p}(r-\zeta)(\eta-1)}{2 r}>0$ holds in the case of $E<b_{p}(\eta+\xi+$ $\left.\frac{(1-(\eta-1) \zeta) R}{r}\right)-b_{p} \frac{(\eta-1)(r-\zeta)(1+R)}{2 r}$.

Therefore, for all $x$ such as $x>\psi_{T 2}$, the following is the case.

$$
\begin{aligned}
x-h_{T}(x) & =\left(1-D h_{T 3}\right)\left(x-\psi_{T 2}\right)-\frac{E}{1+R} \\
& +\left(1-D h_{T 3}\right) \psi_{T 2}>0 .
\end{aligned}
$$

It is easy to check that $\psi_{T *}=h_{T 2}\left(\psi_{T *}\right)$ and $D h_{T 2}<-1$. Thus the 1-D map: $\psi(\tau)=h_{T}(\psi(\tau-1))$ has a unique unstable fixed point $\psi_{T *}(>0)$ in the interval $\left[\psi_{T 1}, \psi_{T 2}\right]$.

In the case of $k=N$ : It is easy to check the following properties: (1) $\psi_{N *}>0$ holds because $r-\zeta>0$ and $r-R \zeta>0$. (2) $\psi_{N *}-\psi_{N 1}=\frac{b_{p}(r-\zeta)(r(1-\xi)-R \zeta)}{2 r(r-R \zeta)}+\frac{E(r-\zeta)}{2(r-R \zeta)}>0$ and $\psi_{N 2}-\psi_{N *}=\frac{(r-\zeta)(\xi r+\eta(r-R \zeta)) b_{p}}{2 r(r-R \zeta)}-\frac{E(r-\zeta)}{2(r-R \zeta)}>0$, i.e., $\psi_{N *} \in\left[\psi_{N 1}, \psi_{N 2}\right]$ hold in the case of $E>b_{p}\left(\xi+\frac{R \zeta-r}{r}\right)$ and $E<b_{p}\left(\xi-\frac{\eta(R \zeta-r)}{r}\right)$.

Furthermore, for all $x$ such as $x<\psi_{N 1},\left(D h_{N 1}-1\right)\left(x-\psi_{N 1}\right)>0$ holds because $\left(D h_{N 1}-1\right)<0$ and $\left(x-\psi_{N 1}\right)<0$ for all $x$ such as $x<\psi_{N 1} \cdot \frac{E}{1+R}+\left(D h_{N 1}-1\right) \psi_{N 1}>0$ holds in the case of $E>b_{p}\left(\xi+\frac{R \zeta-r}{r}\right)$.

Therefore, for all $x$ such as $x<\psi_{N 1}$, the following is the case.

$$
\begin{aligned}
h_{N}(x)-x & =\left(D h_{N 1}-1\right)\left(x-\psi_{N 1}\right)+\frac{E}{1+R} \\
& +\left(D h_{N 1}-1\right) \psi_{N 1}>0 .
\end{aligned}
$$

In the similar way above, for all $x$ such as $x>\psi_{N 2},\left(1-D h_{N 3}\right)\left(x-\psi_{N 2}\right)>0$ holds because $\left(1-D h_{N 3}\right)>0$ and $\left(x-\psi_{N 2}\right)>0$ for all $x$ such as $x>\psi_{N 2} \cdot-\frac{E}{1+R}+(1-$ $\left.D h_{N 3}\right) \psi_{N 2}>0$ holds in the case of $E<b_{p}\left(\xi-\frac{\eta(R \zeta-r)}{r}\right)$.

Therefore, for all $x$ such as $x>\psi_{N 2}$, the following is the case.

$$
\begin{aligned}
x-h_{N}(x) & =\left(1-D h_{N 3}\right)\left(x-\psi_{N 2}\right)-\frac{E}{1+R} \\
& +\left(1-D h_{N 3}\right) \psi_{N 2}>0 .
\end{aligned}
$$


It is easy to check that $\psi_{N *}=h_{N 2}\left(\psi_{N *}\right)$ and $D h_{N 2}<-1$. Thus, the 1-D map $\psi(\tau)=$ $h_{N}(\psi(\tau-1))$ has a unique unstable fixed point $\psi_{N *}(>0)$ in the interval $\left[\psi_{N 1}, \psi_{N 2}\right]$.

Item 1 is proved.

Next, under the conditions that Theorem 2 holds, the following is the case.

In the case of $k=T$, it is easy to check the following properties.

(1) $0<D h_{T 1}<1, D h_{T 2}<-1$, and $0<D h_{T 3}<1$ hold from Corollary 1. (2) $h_{T}\left(\psi_{T 1}\right)>$ $\psi_{T 2}$ because $h_{T}\left(\psi_{T 1}\right)-\psi_{T 2}>0$ holds in the case of $E>b_{p}\left(1+\xi+\frac{R}{r}\right)+b_{p} \frac{(\eta-1)(r-\zeta)(1+R)}{2 r}$. (3) $\psi_{T 1}>h_{T}\left(\psi_{T 2}\right)$ because $\psi_{T 1}-h_{T}\left(\psi_{T 2}\right)>0$ holds in the case of $E<b_{p}(\eta+\xi+$ $\left.\frac{(1-(\eta-1) \zeta) R}{r}\right)-b_{p} \frac{(\eta-1)(r-\zeta)(1+R)}{2 r}$.

In the case of $k=N$, it is easy to check the following properties: (1) $0<D h_{N 1}<1$, $D h_{N 2}<-1$, and $0<D h_{N 3}<1$ hold from Corollary 1. (2) $h_{N}\left(\psi_{N 1}\right)>\psi_{N 2}$ because $h_{N}\left(\psi_{N 1}\right)-\psi_{N 2}>0$ holds in the case of $E>b_{p}\left(\xi+\frac{R \zeta-r}{r}\right)$. (3) $\psi_{N 1}>h_{N}\left(\psi_{N 2}\right)$ because $\psi_{N 1}-h_{N}\left(\psi_{2}\right)>0$ holds in the case of $E<b_{p}\left(\xi-\frac{\eta(R \zeta-r)}{r}\right)$.

Therefore, there exists interval $I_{k \psi}=\left[h_{k}\left(\psi_{k 2}\right), h_{k}\left(\psi_{k 1}\right)\right] \supset\left[\psi_{k 1}, \psi_{k 2}\right]$ such that $h_{k}\left(\psi_{k 2}\right)<$ $\psi_{k 1}<\psi_{k 2}<h_{k}\left(\psi_{k 1}\right)$. Furthermore, in the interval $\left[h_{k}\left(\psi_{k 2}\right), \psi_{k 1}\right] \subset I_{k \psi}, h_{k}$ has a positive slope $D h_{k 1}$ such as $0<D h_{k 1}<1$. In the interval $\left[\psi_{k 1}, \psi_{k 2}\right] \subset I_{k \psi}, h_{k}$ has negative slope $D h_{k 2}$ such as $D h_{k 2}<-1$. In the interval $\left[\psi_{k 2}, h_{T}\left(\psi_{k 1}\right)\right] \subset I_{k \psi}, h_{k}$ has positive slope $D h_{k 3}$ such as $0<D h_{k 3}<1$.

Then, $h_{k}\left(\psi_{k 1}\right)-h_{k}\left(h_{k}\left(\psi_{k 1}\right)\right)=h_{k}\left(\psi_{k 1}\right)-\left(h_{k}\left(\psi_{k 2}\right)+D h_{k 3}\left(h_{k}\left(\psi_{k 1}\right)-\psi_{k 2}\right)\right)>h_{k}\left(\psi_{k 1}\right)-$ $\left(h_{k}\left(\psi_{k 2}\right)+\left(h_{k}\left(\psi_{k 1}\right)-\psi_{k 2}\right)\right)=\psi_{k 2}-h_{k}\left(\psi_{k 2}\right)>0$ holds because $h_{k}\left(h_{k}\left(\psi_{k 1}\right)\right)=h_{k}\left(\psi_{k 2}\right)+$ $D h_{k 3}\left(h_{k}\left(\psi_{k 1}\right)-\psi_{k 2}\right)<h_{k}\left(\psi_{k 2}\right)+\left(h_{k}\left(\psi_{k 1}\right)-\psi_{k 2}\right)$ holds. In the similar way above, $h_{k}\left(h_{k}\left(\psi_{k 2}\right)\right)-h_{k}\left(\psi_{k 2}\right)=h_{k}\left(\psi_{k 1}\right)-D h_{k 1}\left(\psi_{k 1}-h_{k}\left(\psi_{k 2}\right)\right)-h_{k}\left(\psi_{k 2}\right)>h_{k}\left(\psi_{k 1}\right)-\left(\psi_{k 1}-\right.$ $\left.h_{k}\left(\psi_{k 2}\right)\right)-h_{k}\left(\psi_{k 2}\right)=h_{T}\left(\psi_{k 1}\right)-\psi_{k 1}>0$ holds because $h_{k}\left(h_{k}\left(\psi_{k 2}\right)\right)=h_{k}\left(\psi_{k 1}\right)-D h_{k 1}\left(\psi_{k 1}-\right.$ $\left.h_{k}\left(\psi_{k 2}\right)\right)>h_{k}\left(\psi_{k 1}\right)-\left(\psi_{k 1}-h_{k}\left(\psi_{k 2}\right)\right)$ holds. Therefore, it is confirmed that $h_{k}\left(\psi_{k 1}\right)=$ $\max _{x \in I_{k \psi}} h_{k}(x)$ and $h_{k}\left(\psi_{k 2}\right)=\min _{x \in I_{k \psi}} h_{k}(x)$. Thus $h_{T}\left(I_{k \psi}\right) \subset I_{k \psi}$ holds.

Item 2 is proved.

Finally for any $\psi_{0} \in I_{k \psi}$, clearly $h_{k}\left(\psi_{0}\right) \in I_{k \psi}$. for any $\psi_{0} \in\left(-\infty, \psi_{k 1}\right), h_{k}\left(\psi_{0}\right)>\psi_{0}$ from Equation (A1) or Equation (A3). $h_{k}^{j}\left(\psi_{0}\right) \in\left(-\infty, \psi_{k 1}\right), j(>0) \in \mathcal{Z}$ is a monotonically increasing sequence. Therefore, for any $\psi_{0} \in\left(-\infty, \psi_{k 1}\right)$, there exists $j$ th iterate of $h_{k}$ such that $h_{k}^{j}\left(\psi_{0}\right) \in I_{k \psi}, j(>0) \in \mathcal{Z}$. In the similar way for any $\psi_{0} \in\left(\psi_{k 2}, \infty\right), h_{k}\left(\psi_{0}\right)<\psi_{0}$ from Equation (A2) or Equation (A4). $h_{k}^{j}\left(\psi_{0}\right) \in\left(\psi_{k 2}, \infty\right), j(>0) \in \mathcal{Z}$ is a monotonically decreasing sequence. Therefore, for any $\psi_{0} \in\left(\psi_{k 2}, \infty\right)$, there exists $j$ th iterate of $h_{k}$ such that $h_{k}^{j}\left(\psi_{0}\right) \in I_{k \psi}, j(>0) \in \mathcal{Z}$. Item 3 is proved. The proof is now complete.

Proof of Theorem 3. To prove theorem 3, we follow Devaney or Aoki's line for " $\varphi$ has a homoclinic point" implying Definition 1 in $[15,21]$. First of all, we introduce the following definitions, propositions, and notations that are used throughout the proof:

Definition A1 (Devaney [15]). $\Sigma_{2}=\left\{s=\left(s_{0} s_{1} s_{2} \ldots\right) \mid s_{j}=0\right.$ or 1$\}$.

$\Sigma_{2}$ is called the sequence space on the two symbols 0 and 1 . Elements of $\Sigma_{2}$ are infinite sequences of integers, such as $(000 \ldots)$ or $(0101 \ldots)$. We may make $\Sigma_{2}$ into a metric space as follows. For two sequences $s=\left(s_{0} s_{1} s_{2} \ldots\right)$ and $t=\left(t_{0} t_{1} t_{2} \ldots\right)$, define the distance between them by $d(s, t)=\sum_{i=0}^{\infty} \frac{\left|s_{i}-t_{i}\right|}{2^{i}}$. Since $\left|s_{i}-t_{i}\right|$ is either 0 or 1 , this infinite series is dominated by the geometric series $\sum_{i=0}^{\infty} \frac{1}{2^{i}}=2$; therefore, it converges.

Definition A2 (Devaney [15]). The shift map $\sigma: \Sigma_{2} \rightarrow \Sigma_{2}$ is given by $\sigma\left(s_{0} s_{1} s_{2} \ldots\right)=$ $\left(s_{1} s_{2} s_{3} \ldots\right)$.

The shift map simply "forgets" the first entry in a sequence, and shifts all other entries one place to the left. Clearly, $\sigma$ is a two-to-one map of $\Sigma_{2}$, as $s_{0}$ may be either 0 or 1 . Moreover, in the metric defined above, $\sigma$ is a continuous map. 
Proposition A1 (Devaney [15]). $d(s, t)$ is a metric on $\Sigma_{2}$.

Proposition A2 (Devaney [15]). Let $s, t \in \Sigma_{2}$ and suppose $s_{i}=t_{i}$ for $i=0,1, \ldots, n$. Then, $d(s, t) \leq \frac{1}{2^{n}}$. Conversely, if $d(s, t)<\frac{1}{2^{n}}$, then $s_{i}=t_{i}$ for $i=0,1, \ldots, n$.

Proposition A3 (Devaney [15]). $\sigma: \Sigma_{2} \rightarrow \Sigma_{2}$ is continuous.

Definition A3 (Devaney [15]). The itinerary of $x$ is a sequence $S(x)=s_{0} s_{1} s_{2} \ldots$ where $s_{j}=0$ if $f^{j}(x) \in I_{0}, s_{j}=1$, if, $f^{j}(x) \in I_{1}$.

Thus, the itinerary of $x$ is an infinite sequence of 0 s and 1 s. That is, $S(x)$ is a point in the sequence space $\Sigma_{2}$. We think of $S$ as a map from $\Lambda$ to $\Sigma_{2}$. This map has several interesting properties.

Let $J \subseteq I_{0}$ or $J \subseteq I_{1}$ be a closed interval. Let $f^{-n}(J)=\left\{x \in I_{0} \cup I_{1} \mid f^{n}(x) \in J, n \geq 1\right\}$. $f^{0}(J)=J$. Since item $A 1$ above holds, $f^{-n}(J)$ exists as the preimage of $J$ under functions $f^{n}(x)$.

Let $I_{s_{0} s_{1} \ldots s_{n}}=\cap_{0 \leq k \leq n} f^{-k}\left(I_{s_{k}}\right)$

$=\cap_{0 \leq k \leq n}\left(f^{k}\left(I_{0} \cup I_{1}\right) \cap I_{s_{k}}\right) \quad=\left\{x \in I_{0} \cup I_{1} \mid x \in I_{s_{0}}, f(x) \in I_{s_{1}}, f^{2}(x) \in I_{s_{2}}, \ldots, f^{n}(x)\right.$ $\left.\in I_{s_{n}}\right\}=\bar{I}_{s_{0}} \cap f^{-1}\left(I_{s_{1}}\right) \cap \ldots \cap f^{-n}\left(I_{s_{n}}\right)=I_{s_{0}} \cap\left(f\left(I_{0} \cup I_{1}\right) \cap I_{s_{1}}\right) \cap \ldots \cap\left(f^{n}\left(I_{0} \cup I_{1}\right) \cap I_{s_{n}}\right)$.

$f^{-1}(J)$ consists of two closed subintervals, one in $I_{0}$ and one in $I_{1}$. Hence, $f^{-1}\left(I_{0} \cup I_{1}\right)$ consists of four disjoint closed subintervals, two in $I_{0}$ and two in $I_{1}$. Similarly, in general, $f^{-n}(J)$ consists of $2^{n}$ closed subintervals, $2^{n-1}$ in $I_{0}$ and $2^{n-1}$ in $I_{1}$. Hence, $f^{-n}\left(I_{0} \cup I_{1}\right)$ consists of $2^{n+1}$ disjoint closed subintervals, $2^{n}$ in $I_{0}$ and $2^{n}$ in $I_{1}$. Then, the sequence $I_{s_{0} s_{1} \ldots s_{n}}$ is not empty. We have $\cap_{0 \leq k \leq n} f^{-k}\left(I_{0} \cup I_{1}\right)=\cap_{0 \leq k \leq n}\left(f^{k}\left(I_{0} \cup I_{1}\right) \cap\left(I_{0} \cup I_{1}\right)\right)=$ $\cap_{0 \leq k \leq n} f^{k}\left(I_{0} \cup I_{1}\right)=\cup_{s_{0} s_{1} \ldots s_{n}=0,1} I_{s_{0} s_{1} \ldots s_{n}}$. First, we consider the properties of the sequence $I_{s_{0} s_{1} \ldots s_{n}}$. Note that $I_{s_{0} s_{1} \ldots s_{n}}=I_{s_{0}} \cap f^{-1}\left(I_{s_{1} s_{2} \ldots s_{n}}\right)$ and $I_{s_{0} s_{1} \ldots s_{n}}=I_{s_{0} s_{1} \ldots s_{n-1}} \cap f^{-n}\left(I_{s_{n}}\right) \subset$ $I_{s_{0} s_{1} \ldots s_{n-1}}$ hold. We may assume that $I_{s_{1} s_{2} \ldots s_{n}}$ is a nonempty subinterval so that, by the observation above, $f^{-1}\left(I_{s_{1} s_{2} \ldots s_{n}}\right)$ consists of two closed intervals, one in $I_{0}$ and one in $I_{1}$. Hence, $I_{s_{0}} \cap f^{-1}\left(I_{s_{1} s_{2} \ldots s_{n}}\right)$ is a single closed interval. $I_{s_{0} s_{1} \ldots s_{n}}$ forms a nested sequence of nonempty closed intervals because $I_{s_{0} s_{1} \ldots s_{n}}=I_{s_{0} s_{1} \ldots s_{n-1}} \cap f^{-1}\left(I_{s_{n}}\right) \subset I_{s_{0} s_{1} \ldots s_{n-1}}$.

Therefore, we conclude that $\cap_{0 \leq n \leq \infty} I_{s_{0} s_{1} \ldots s_{n}}$ is nonempty closed, set and $\Lambda$ is also nonempty closed set such that $\Lambda=\cap_{0 \leq n \leq \infty} f^{n}\left(I_{0} \cup I_{1}\right)=\cap_{0 \leq n \leq \infty} f^{-n}\left(I_{0} \cup I_{1}\right)=\cup_{s_{0} s_{1} \ldots=0,1}$ $\left(\cap_{0 \leq n \leq \infty} I_{s_{0} s_{1} \ldots s_{n}}\right)$.

Secondly, we show that $S$ is one to one. Let $x, y \in \Lambda$ and suppose $S(x)=S(y)$. Then, for each $n, f^{n}(x)$ and $f^{n}(y)$ lie on the same interval $I_{0}$ or $I_{1}$. This implies that $f$ is monotone on the interval between $f^{n}(x)$ and $f^{n}(y)$. Consequently, all points in this interval remain in $I_{0} \cup I_{1}$ when we apply $f$. Now, $|D f|>K>1$ at all points in this interval; thus, as in item $A 2$, each iteration of $f$ expands this interval by a factor of $K$. Hence, the distance between $f^{n}(x)$ and $f^{n}(y)$ grows without bound, so these two points $f^{n}(x)$ and $f^{n}(y)$ must eventually lie on $I_{0}$ and $I_{1}$ (or $I_{1}$ and $I_{0}$ ), respectively. This contradicts the fact that they have the same itinerary.

Thirdly, to see that $S$ is onto, let $s=\left(s_{0} s_{1} s_{1} \ldots\right)$. We must produce $x \in \Lambda$ with $S(x)=s$. Note that if $x \in \cap_{0 \leq n \leq \infty} I_{S_{0} s_{1} \ldots s_{n}}$, then $x \in I_{S_{0}}, f(x) \in I_{S_{1}}$, etc. Hence, there exists $x \in \Lambda$ such that $S(x)=\left(s_{0} s_{1} s_{1} \ldots\right)$. This proves that $S$ is onto. Observe that $x \in \cap_{0 \leq n \leq \infty} I_{s_{0} s_{1} \ldots s_{n}}$ consists of a unique point because $S$ is one to one. In particular, we have that diameter of $I_{s_{0} s_{1} \ldots s_{n}}$ tending to 0 as $n \rightarrow \infty$.

Furthermore, to prove the continuity of $S$, we choose $x \in \Lambda$ and suppose that $S(x)=\left(s_{0} s_{1} s_{2} \ldots\right)$. Let $\epsilon>0$. Pick $n$ so that $1 / 2^{n}<\epsilon$. Consider the closed subintervals $I_{t_{0}} t_{1} \ldots t_{n}$ defined above for all possible combinations $t_{0} t_{1} \ldots t_{n}$. These subintervals are all disjoint, and $\Lambda$ is contained in their union. These are $2^{n+1}$ such intervals, and $I_{S_{0} S_{1} \ldots s_{n}}$ is one of them. Hence, we may choose $\delta$ such that $|x-y|<\delta$ and $y \in \Lambda$ implies that $y \in I_{S_{0} S_{1} \ldots s_{n}}$. Therefore, $S(y)$ agrees with $S(x)$ in the first $n+1$ terms. Thus, by Proposition A2, we have $d(S(x), S(y)) \leq \frac{1}{2^{n}}<\epsilon$. This proves the continuity of $S$. Since $\Lambda$ is compact (bounded closed sets) and $S$ is continuous and one to one, $S^{-1}$ is also continuous. Thus, $S$ is a homeomorphism. 
Finally, to prove that $S \circ f=\sigma \circ S$, i.e., $S$ provides an equivalence between the dynamics of $f$ on $\Lambda$ and $\sigma$ on $\Sigma_{2}$. Let $x_{0} \in \Lambda$ and suppose that $S\left(x_{0}\right)=\left(s_{0} s_{1} s_{2} \ldots\right)$. Then, we have $x_{0} \in I_{s_{0}}, f\left(x_{0}\right) \in I_{s_{1}}, f^{2}\left(x_{0}\right) \in I_{s_{2}}$ and so forth. However, the fact that $f\left(x_{0}\right) \in$ $I_{s_{1}}, f^{2}\left(x_{0}\right) \in I_{s_{2}}$, etc., says that $S\left(f\left(x_{0}\right)\right)=\left(s_{1} s_{2} s_{3} \ldots\right)$, so $S\left(f\left(x_{0}\right)\right)=\sigma\left(S\left(x_{0}\right)\right)$, which is what we wanted to prove. The proof is now complete.

Proof of Theorem 4 . Let $\mathcal{R}$ be the set of real numbers. Under the conditions that Theorem 4 holds, it is easy to check the following properties: (1) the break points $-\frac{\left(k_{1}-n_{1}\right) k_{1}}{n_{1}-1}(<0)$ and $-\frac{\left(k_{2}-n_{1}\right) k_{1}}{n_{1}-1}(<0)$ are given by the solutions of $\left\{x \in \mathcal{R} \mid H_{1}(x)=k_{1}\right\}$ and $\{x \in$ $\left.\mathcal{R} \mid H_{1}(x)=k_{2}\right\}$, respectively. (2) The break points of $H\left(x_{n}\right): k_{1}$ and $k_{2}$ are also the break points of $H^{2}\left(x_{n}\right)$. (3)The break points $k_{2}-k_{2}\left(k_{2}-k_{1}\right)$ and $k_{2}\left(1-k_{1}\right)+k_{1}^{2}$ are given by the solutions of $\left\{x \in \mathcal{R} \mid H_{2}(x)=k_{2}\right\}$ and $\left\{x \in \mathcal{R} \mid H_{2}(x)=k_{1}\right\}$, respectively. (4) $0<k_{1}<k_{2}-k_{2}\left(k_{2}-k_{1}\right)<k_{2}\left(1-k_{1}\right)+k_{1}^{2}<k_{2}$ holds because $k_{2}-\left(k_{2}(1-\right.$ $\left.\left.k_{1}\right)+k_{1}^{2}\right)=k_{1}\left(k_{2}-k_{1}\right)>0, k_{2}\left(1-k_{1}\right)+k_{1}^{2}-\left(k_{2}-k_{2}\left(k_{2}-k_{1}\right)\right)=\left(k_{2}-k_{1}\right)^{2}>0$ and $k_{2}-k_{2}\left(k_{2}-k_{1}\right)-k_{1}=\left(1-k_{2}\right)\left(k_{2}-k_{1}\right)>0$ hold. (5) The break points $k_{2}+\frac{k_{1}\left(1-k_{2}\right)}{n_{2}}$ and $k_{2}+\frac{k_{2}\left(1-k_{2}\right)}{n_{2}}$ are given by the solutions of $\left\{x \in \mathcal{R} \mid H_{3}(x)=k_{1}\right\}$ and $\left\{x \in \mathcal{R} \mid H_{3}(x)=k_{2}\right\}$, respectively. (6) $k_{2}<k_{2}+\frac{k_{1}\left(1-k_{2}\right)}{n_{2}}<1<k_{2}+\frac{k_{2}\left(1-k_{2}\right)}{n_{2}}$ holds because $k_{2}+\frac{k_{2}\left(1-k_{2}\right)}{n_{2}}-1=$ $\left(1-k_{2}\right)\left(\frac{k_{2}}{n_{2}}-1\right)>0$ and $1-\left(k_{2}+\frac{k_{1}\left(1-k_{2}\right)}{n_{2}}\right)=\left(1-k_{2}\right)\left(1-\frac{k_{1}}{n_{2}}\right)>0$ hold.

Furthermore, $\left|D H_{1} H_{1}\right|<1$ because $0<\left(1-n_{1}\right), k_{1}<1$ and $1-n_{1}<k_{1}$. $\left|D H_{2} H_{2}\right|>$ 1 because $0<k_{1}, k_{2}<1, k_{1}<k_{2}$, and $0<k_{2}-k_{1}<1$. $\left|D H_{3} H_{3}\right|<1$ because $0<$ $\left(1-k_{2}\right), n_{2}<1$ and $k_{2}<1-n_{2} .\left|D H_{3} H_{1}\right|=\left|D H_{1} H_{3}\right|<1$ because $1-n_{1}<k_{1}$ and $k_{2}<1-n_{2} .\left|D H_{3} H_{2}\right|=\left|D H_{2} H_{3}\right|>1$ because $0<k_{2}-k_{1}<1$, and $n_{2}>\left(k_{2}-k_{1}\right)\left(1-k_{2}\right)$. $\left|D H_{1} H_{2}\right|=\left|D H_{2} H_{1}\right|>1$ because $0<k_{2}-k_{1}<1$ and $1-k_{1}\left(k_{2}-k_{1}\right)>n_{1}$. The proof is now complete.

Proof of Theorem 5. Under the conditions of Theorem 5, Theorem 4 holds. In the following, $H^{2}(\Psi)$ is given by the map of Theorem 4. Then $H^{2}\left(k_{2}-k_{2}\left(k_{2}-k_{1}\right)\right)=0$, $H^{2}\left(k_{2}\left(1-k_{2}\right)+k_{1}^{2}\right)=1$, and $[0,1]=\left\{\Psi \mid H^{2}(\Psi)=H_{2} H_{2}(\Psi), \Psi \in I_{0}\right\}$. Therefore, clearly $I_{0} \cup I_{1} \subset H^{2}\left(I_{0}\right) .\left|D H^{2}(\Psi)\right|>1$ also holds for $\Psi \in I_{0}$ because $\left|D H^{2}(\Psi)\right|=\left|D H_{2} H_{2}(\Psi)\right|>$ 1. $H^{2}\left(k_{2}+\frac{k_{1}\left(1-k_{2}\right)}{n_{2}}\right)=1, H^{2}(1)=\frac{k_{2}-n_{2}}{k_{2}-k_{1}}$, and $\left[\frac{k_{2}-n_{2}}{k_{2}-k_{1}}, 1\right]=\left\{\Psi \mid H^{2}(\Psi)=H_{2} H_{3}(\Psi), \Psi \in I_{1}\right\}$. Therefore, $I_{0} \cup I_{1} \subset H^{2}\left(I_{1}\right)$ holds because $k_{2}-k_{2}\left(k_{2}-k_{1}\right)>\frac{k_{2}-n_{2}}{k_{2}-k_{1}}$ holds. $\left|D H^{2}(\Psi)\right|>1$ also holds for $\Psi \in I_{1}$ because $\left|D H^{2}(\Psi)\right|=\left|D H_{2} H_{3}(\Psi)\right|>1$. Thus, item 1 is proved.

Next to prove the item 2, it is sufficient that $H^{2}$ is considered as the map $f$ in Theorem 3 . Under the item 1 condition, $H^{2}$ is satisfied with the condition of Theorem 3 . The item 2 is also proved. The proof is now complete.

\section{References}

1. Saito, T. A Chaos Generator Based on a Quasi-Harmonic Oscillator. IEEE Trans. Circuits Syst. 1985, 32, 320-331. [CrossRef]

2. Saito, T.; Nakagawa, S. Chaos from a hysteresis and switched circuit. Philos. Trans. R. Soc. Lond. Math Phys. Sci. 1995, 352, 47-57.

3. Miyoshi, T.; Saito, T.; Inaba, N. Chaotic Phenomena in a Iron Resonant Circuit Including an Inductor with Complete Saturation Characteristic. IEICE Trans. 1997, 80, 346-354. (In Japanese)

4. Kennedy, M.P.; Rovatti, R.; Setti, G. (Eds.) Chaotic Electronics in Telecommunications; CRC Press: Boca Raton, FL, USA, 2000.

5. Nagumo, J.; Shimura, M. Self-oscillation in a transmission line with a tunnel diode. Proc. IRE. 1961, 49, 1281-1291. [CrossRef]

6. Nagumo, J.; Sato, S. On a Response Characteristic of a Mathematical Neuron Model. Kybernetik 1972, 10, 155-164. (In Japanese) [CrossRef] [PubMed]

7. Nakano, H.; Okazaki, H. Bifurcation Phenomena of a Distributed parameter System with a Nonlinear Element Having Negative Resistance. IEICE Trans. Fundam. 1992, 75, 339-346.

8. Sharkovsky, A.N.; Maistrenko, Y.U.; Deregel, P.H.; Chua, L.O. Dry Turbulence a Time-Delayed Chua's Circuit. J. Circuits Syst. Comput. 1993, 3, 645-668. [CrossRef]

9. Sharkovsky, A.N. Chaos from a Time-Delayed Chua's Circuit. IEEE Trans. Circuits Syst. 1993, 40, 781-783. [CrossRef]

10. Sharkovsky, A.N.; Romanenko, E.; Berezovsky, S. Ideal turbulence: Definition and models. In Proceedings of the 2003 IEEE International Workshop on Workload Characterization (IEEE Cat. No.03EX775), St. Petersburg, Russia, 20-22 August 2003; Volume 1, pp. 23-30. 
11. Miyabayashi, N.; Moro, S.; Mori, S. Spatio-temporal dynamics in an array of time delayed Van der Pol oscillators. In Proceedings of the 1997 IEEE International Symposium on Circuits and Systems (ISCAS '97), Hong Kong, China, 12 June 1997; Volume 2, pp. 917-920.

12. Bucolo, M.; Buscarino, A.; Famoso, C.; Fortuna, L.; Gagliano, S. Imperfections in Integrated Devices Allow the Emergence of Unexpected Strange Attractors in Electronic Circuits. IEEE Access 2021, 9, 29573-29583. [CrossRef]

13. Kohda, T. Discrete Dynamics and Chaos; Corona Publishing: Tokyo, Japan, 1998. (In Japanese)

14. Guckenheimer, J.; Holmes, P. Nonlinear Oscillations Dynamical Systems, and Bifurcation of Vector Fields; Springer: New York, NY, USA, 1983.

15. Devaney, R.L. An Introduction to Chaotic Dynamical Systems; The Benjamin/Cummings Publishing: San Francisco, CA, USA, 1986.

16. Robinson, C. Dynamical Systems, Stability, Symbolic Dynamics, and Chaos, 2nd ed.; CRC Press: Boca Raton, FL, USA, 1999.

17. Kokubu, H. Foundation of Dynamical Systems; Asakura Publishing: Tokyo, Japan, 2000. (In Japanese)

18. Thunberg, H. Periodicity versus Chaos in One-Dimensional Dynamics. SIAM Rev. 2001, 43, 3-30. [CrossRef]

19. Moser, J. Stable and Random Motions in Dynamical Systems; Princeton University Press: Princeton, NJ, USA, 1973.

20. Oono, Y.; Oshikawa, M. Chaos in Nonlinear Difference Equations I. Prog. Theor. Phys. 1980, 64, 54-67. [CrossRef]

21. Aoki, N. Dynamical Systems and Chaos-Geometric Construction of Nonlinear Phenomena; Kyoritsu Publishing: Tokyo, Japan, 2000. (In Japanese)

22. Okazaki, H.; Okazaki, C.; Honda, H.; Nakano, H. Rigorous verification of formal chaos produced by one-dimensional discrete dynamical system with use of interval arithmetic. In Proceedings of the 2005 IEEE 48th Midwest Symposium on Circuits and Systems (The IEEE MWSCAS 2005), Covington, KY, USA, 7-10 August 2005; pp. 1597-1660.

23. Lynch, S. Dynamical Systems with Applications Using MAPLE; Birkhäuser: Basel, Switzerland, 2010. 